\title{
Determinant Bundles, Manifolds with Boundary and Surgery
}

\author{
Paolo Piazza \\ Istituto Matematico "G. Castelnuovo", Università degli Studi di Roma "La Sapienza", P. le Aldo \\ Moro 2, I-00185 Roma, Italy. E-mail address: piazza@mat.uniromal.it
}

Received: 28 August 1995 / Accepted: 30 October 1995

\begin{abstract}
We define determinant bundles associated to the following data: (i) a family of generalized Dirac operators on even dimensional manifolds with boundary, (ii) the choice of a spectral section for the family of Dirac operators induced on the boundary. Under the assumption that the operators of the boundary family have null spaces of constant dimension we define, through the notion of $b$-zeta function, a Quillen metric. We also introduce the analogue of the Bismut-Freed connection. We prove that the curvature of a natural perturbation of the BismutFreed connection equals the 2 -form piece in the right-hand side of the family index formula, thus extending to manifolds with boundary results of Quillen, Bismut and Freed. Given a closed fibration, we investigate the behaviour of the Quillen metric and of the Bismut-Freed connection under the operation of surgery along a fibering hypersurface. We prove, in particular, additivity formulae for the curvature and for the logarithm of the holonomy.
\end{abstract}

\section{Introduction}

Determinants of elliptic operators arise frequently in Quantum Field Theory. In the evaluation of path integrals over families of Dirac operators $\delta=\left(\delta_{z}\right), z \in B$, it is desirable to have a determinant function, DET: $B \rightarrow \mathbb{C}$, assigning a complex number to each operator $\partial_{z}$.

As explained in the fundamental work of Quillen [23] in general it is only possible to assign a determinant line bundle $\operatorname{det}(ð)$ and a natural section $\sigma \in$ $\mathscr{C}^{\infty}(B ; \operatorname{det}(ð))$ to any such Dirac family. If the determinant line bundle is trivial then by fixing a trivializing section $\tau \in \mathscr{C}^{\infty}(B$; $\operatorname{det}(ð))$ we can define a determinant function $\operatorname{DET}_{\tau} \in \mathscr{C}^{\infty}(B)$ by comparing the two sections $\sigma, \tau: \sigma(z)=\operatorname{DET}_{\tau}(z) \tau(z)$. In the physics literature the obstruction to find such a trivializing section is referred to as an anomaly. Even if the determinant line bundle is trivial there are many possible choices of $\tau$ and it is natural to try to determine a canonical one.

In the case of $\bar{\partial}$-operators acting on a vector bundle $E$ over a Riemann surface $M$, thus with parameter space $B$ equal to the space $\mathscr{A}$ of holomorphic structures 
on $E$, Quillen solved this problem by introducing a natural metric $\|\cdot\|_{Q}$ on $\operatorname{det}(\bar{\partial})$ and computing the curvature of the unique complex metric-compatible connection $\nabla^{\text {det }}$. The curvature formula allows for the construction of a new metric with flat associated complex metric-compatible connection. Once such a connection has been constructed the determinant function is obtained as above by fixing an everywhere flat (trivializing) section which exists since the space $\mathscr{A}$ is contractible.

Bismut and Freed $[10,11]$ extended the work of Quillen to an arbitrary smooth family of twisted Dirac operators $\partial$ on even-dimensional manifolds by introducing the Quillen metric on $\operatorname{det}(ð)$ together with a natural metric-compatible connection. Building also on conjectures of Witten [27] they proved formulae for the curvature and the holonomy of this connection. These formulae represent the obstruction to trivializing the determinant bundle by parallel transport, as done by Quillen, and they are often referred to as the local and global anomaly formulae. The global anomaly formula was also proved, independently, by Cheeger in [13]. See also [14,26]. In this paper we have two specific goals in mind. First we extend to manifolds with boundary the results of Bismut and Freed, with the notable exception of the global anomaly formula which will be the object of a future publication. Second we give surgery results (or, in a more physical language, sewing rules) for the local and global anomalies whenever a fibration $\psi: M \rightarrow B$ of closed manifolds defining a family of generalized Dirac operators $\partial$ is cut along a fibering hypersurface $H$, producing two families of generalized Dirac operators on manifolds with boundary.

Let us describe more precisely the contents of this paper. Families of Dirac operators $\partial$ on spin manifolds with boundary were first considered in [6]. Much of the analysis carried out there is done under the assumption that the family of Dirac operators induced on the boundary, $\partial_{0}$, is invertible; we shall refer to this as the invertible case. In [18] (see also [19] for the odd-dimensional case) the main results of [6] are extended to an arbitrary family of Dirac operators, i.e. with no assumption on the boundary family. Given such a family $ð$, we can always choose a spectral section $P$ for the boundary family $\partial_{0}$ (see Sect. 1 below for the definition) and thus a smooth family of generalized Atiyah-Patodi-Singer boundary conditions. In [18] an index formula for the Chern character of the index bundle associated to these generalized Atiyah-Patodi-Singer boundary value problems is proved. To prove such a formula the $b$-calculus for elliptic operators on manifolds with boundary is used in a crucial way. First of all it allows for a deformation of the family of generalized Atiyah-Patodi-Singer boundary value problems $(ð, P)$ defined by our data to a family of perturbed Dirac operators $\partial+A_{P}$, with $A_{P}$ a family of $b$-pseudodifferential operators of order $-\infty$, with the property that the boundary family $ð_{0}+A_{P, 0}$ is invertible. The ordinary Atiyah-Patodi-Singer boundary condition, suitably interpreted in the framework of $b$-geometry, can be applied in this case, giving a continuous family of Fredholm operators. Since the two families of Fredholm operators so defined, viz. $(ð, P)$ and $ð+A_{P}$, are homotopic, they fix the same index class in $K^{0}$ of the base. Thus one is reduced to proving the family index theorem in the invertible case but for a larger class of operators. The $b$-calculus is used again at this point, in the form of a formula for the $b$-trace of a supercommutator, to compute explicitly the boundary contribution, a generalized eta-form, which enters into the final formula for the Chern character. By applying these results of [18] we are able to give in Sect. 1 the general definition of determinant bundle for families of Dirac operators on manifolds with boundary, together with an explicit formula for its Chern class. The definition depends on the choice of the spectral section $P$, although there is a formula relating the Chern class of 
two determinant bundles associated to different choices of spectral sections. Using this formula we show that there always exists a spectral section $Q$ for which the determinant bundle is trivial. We use the notation $\operatorname{det}_{b}(ð, P)$ for the determinant line bundle associated to $\partial$ and the spectral section $P$.

Next we pass to the study of the Hermitian geometry of such a determinant line bundle. To do so we need to assume the operators of the boundary family to have null space of constant dimension in $z \in B$. We refer to this as the constant rank case. We then choose $P$ to be equal to $\Pi_{\geqq}$, the spectral projection corresponding to the non-negative eigenvalues of the boundary family. Although all of our results are valid under the constant rank assumption we start in Sects. 2, 3 to treat the invertible case. Using the regularity results for the $b$-zeta function of an elliptic $b$-differential operator $([22,17])$ we introduce in Sect. 2 the $b$-Quillen metric on $\operatorname{det}_{b}\left(ð, \Pi_{\geqq}\right)$. We also study the variation of the $b$-zeta function and of its derivative in $0 \in \mathbb{C}$. We show in particular that the usual formulae hold. This is not obvious, since by applying Duhamel's principle, boundary contributions arise from the formula for the $b$-trace of a commutator of two operators; we show that these boundary contributions are always zero. The validity of these variation formulae shows once again ([22]) that as far as index theory on manifolds with boundary is concerned, $b$-zeta functions play the same role as ordinary zeta functions in index theory on closed manifolds. In Sect. 3 we use these results to introduce a natural connection ${ }^{b} \nabla^{\text {det }}$. This is the analogue of the Bismut-Freed connection. We prove that this connection is compatible with the $b$-Quillen metric. The point here is again to make sure that various boundary contributions arising from applications of Duhamel's principle are identically zero and that the arguments given in the proof of the compatibility in the closed case (see for example [2]) can be carried over.

In Sect. 4 we investigate the behaviour of the determinant line bundle, the Quillen metric and the Bismut-Freed connection under surgery. The operation of surgery is explained at length at the beginning of that section. To describe it informally consider a fibration of (for simplicity spin) closed manifolds $\psi: M \rightarrow B$ defining a family of Dirac operators $\partial$ as in $[10,11]$. Let $H$ be an embedded disconnecting codimension one submanifold of $M$. We assume that $H$ fibres as well, thus $\psi_{H}: H \rightarrow B . M$ is therefore the union of two fibrations with boundary $M^{0}, M^{1}$ along the fibration $H$, each fibre $\psi^{-1}(z) \equiv M_{z}$ being the union along $H_{z}$ of two manifolds with boundary $M_{z}^{0}, M_{z}^{1}: M_{z}=M_{z}^{0} \bigcup_{H_{z}} M_{z}^{1}$. Let $U_{H} \cong[-1,1] \times H$ be a collar neighbourhood of $H$; by surgery, following [16], we mean the stretching of the collar neighbourhood $U_{H}$ to a cylinder of infinite length. If $g_{M / B}$ is the family of metrics of the spin fibration $\psi: M \rightarrow B$ and if $x \in \mathscr{C}^{\infty}(M)$ is a defining function for $H$, this intuitive idea of stretching is rigorously realized by considering the family of metrics

$$
g_{M / B}(\varepsilon)=\frac{|d x|^{2}}{x^{2}+\varepsilon^{2}}+g_{M / B}
$$

and letting $\varepsilon \downarrow 0$. As $\varepsilon \downarrow 0$ each manifold $M_{z}$ develops a neck $|2 \log \varepsilon|$-long around the hypersurface $H$. For $\varepsilon=0$ we obtain $\bar{M}$, the disjoint union of two exact $b$-fibrations $\bar{M}^{0}, \bar{M}^{1}$ of the sort considered in the first three sections of the paper and the idea is to follow what happens to $\operatorname{det}(ð(\varepsilon))$, its Quillen metric and Bismut-Freed connection when $\varepsilon \downarrow 0$. Under the assumption that the Dirac operators induced on $H_{z}$ are invertible we prove that there exists a natural explicit isomorphism

$$
\operatorname{det}(ð(\varepsilon)) \leftrightarrow \operatorname{det}_{b}\left(ð_{\bar{M}^{0}}, \Pi_{<}\right) \otimes \operatorname{det}_{b}\left({\varlimsup_{\bar{M}}}^{1}, \Pi_{\geqq}\right)
$$


for each $\varepsilon$ small enough. Here $\Pi_{\geqq}$is the spectral projection corresponding to the non-negative eigenvalues of the boundary operator associated to ${\delta_{\bar{M}}}^{1}$. In particular, since $\varnothing$ and $\partial(\varepsilon)$ are homotopic, we obtain an isomorphism

$$
\operatorname{det}(ð) \leftrightarrow \operatorname{det}_{b}\left(\partial_{\bar{M}^{0}}, \Pi_{<}\right) \otimes \operatorname{det}_{b}\left({\varlimsup_{\bar{M}}}^{1}, \Pi_{\geqq}\right) \text {. }
$$

Next we analyze the behaviour of the Quillen metric $\|\cdot\|_{Q(\varepsilon)}$ and of the BismutFreed connection $\nabla^{\text {det, } \varepsilon}$ showing on the one hand that the pushforward under the above isomorphism of the Quillen metric $\|\cdot\|_{Q(\varepsilon)}$ multiplied by $\varepsilon^{\zeta^{\prime}\left(0, \partial_{H}^{2}\right)}$ converges as $\varepsilon \downarrow 0$ to the $b$-Quillen metric on $\operatorname{det}_{b}\left(\partial_{\bar{M}^{0}}, \Pi_{<}\right) \otimes \operatorname{det}_{b}\left(\partial_{\bar{M}^{1}}, \Pi_{\geqq}\right)$whereas the pushforward of the connection $\nabla^{\mathrm{det}, \varepsilon}+\log \varepsilon \cdot d \zeta^{\prime}\left(0, \partial_{H}^{2}\right)$ converges to the BismutFreed connection ${ }^{b} \nabla^{\text {det, } 0} \otimes \mathrm{Id}+\mathrm{Id} \otimes{ }^{b} \nabla^{\text {det, } 1}$. These results yield immediately, in Sect. 5, the (asymptotic) additivity of the curvature and of the logarithm of the holonomy under surgery : as $\varepsilon \downarrow 0$,

$$
\begin{gathered}
\left(\nabla^{\operatorname{det}, \varepsilon}\right)^{2} \rightarrow\left({ }^{b} \nabla^{\text {det, } 0}\right)^{2}+\left({ }^{b} \nabla^{\text {det, } 1}\right)^{2}, \\
\operatorname{hol}_{\gamma}\left(\nabla^{\operatorname{det}, \varepsilon}\right) \rightarrow \operatorname{hol}_{\gamma}\left({ }^{b} \nabla^{\operatorname{det}, 0}\right) \cdot \operatorname{hol}_{\gamma}\left({ }^{b} \nabla^{\text {det, }, 1}\right) \quad \forall \gamma \in \operatorname{Map}\left(\mathbb{S}^{1}, B\right) .
\end{gathered}
$$

These are the sewing rules called for in [25]. They tell us that the two anomalies associated to the fibration $M=M_{0} \bigcup_{H} M_{1}$ can be split into two contributions coming from $M_{0}$ and $M_{1}$. The results of these two sections are based on the pseudodifferential surgery calculus of [16].

In Sect. 6 we finally prove the local anomaly formula on manifolds with boundary in the invertible case which is at this point a simple application of the transgressed form of the family index formula: if $P=\Pi_{\geqq}$, so that the index bundle is simply realized by a family of $b$-differential operators acting on unweighted Sobolev spaces, and if null $\left(\partial^{+}\right) \oplus \operatorname{null}\left(\delta^{-}\right)$is a smooth bundle, so that $\operatorname{Ind}\left(ð, \Pi_{\geqq}\right) \equiv$ $\operatorname{Ind}(ð)=\operatorname{null}(ð)$, then

$$
\operatorname{Ch}(\operatorname{Ind}(ð))=\frac{1}{(2 \pi i)^{\frac{n}{2}}} \int_{M / B} \widehat{A}(M / B) \mathrm{Ch}^{\prime}(E)-\frac{1}{2} \widehat{\eta}+d \int_{0}^{\infty} b-\mathrm{STr}\left(\frac{d \mathbb{A}_{s}}{d s} e^{-A_{s}^{2}}\right) d s
$$

as differential forms on $B$. In this formula $\mathbb{A}_{s}$ is the rescaled Bismut superconnection and $\hat{\eta}$ is the eta form introduced by Bismut and Cheeger (see [6 or 18] for the definition). The Chern character on the left-hand side is computed with respect to the compression of the 1 -form piece of the Bismut superconnection onto the null bundle null(ð). Using this formula and the results of Sects. 2 and 3, the local formula for the curvature of the Bismut-Freed connection on $\operatorname{det}_{b}\left(ð, \Pi_{\geqq}\right)$,

$$
\left({ }^{b} \nabla^{\mathrm{det}}\right)^{2}=\left(\frac{1}{(2 \pi i)^{\frac{n}{2}}} \int_{M / B} \widehat{A}(M / B) \mathrm{Ch}^{\prime}(E)-\frac{1}{2} \widehat{\eta}\right)_{[2]}
$$

follows easily.

The results on surgery and on the local anomaly formula are extended to the constant rank case in Sect. 8. After some preliminary results on the analysis of elliptic families on manifolds with boundary, which are gathered in Sect. 3, we show that for a natural perturbation $\left({ }^{b} \widetilde{\nabla}^{\text {det }}\right)$ of the Bismut-Freed connection on $\operatorname{det}_{b}\left(ð, \Pi_{\geqq}\right)$, the following local anomaly formula holds:

$$
\left({ }^{b} \widetilde{\nabla}^{\mathrm{det}}\right)^{2}=\left(\frac{1}{(2 \pi i)^{\frac{n}{2}}} \int_{M / B} \widehat{A}(M / B) \mathrm{Ch}^{\prime}(E)-\frac{1}{2} \widehat{\eta}-\frac{1}{2} \operatorname{Ch}\left(\text { null } ð_{0}, \nabla^{\text {null }}\right)\right)_{[2]}
$$


with $\nabla^{\text {null }}$ denoting the compression of the 1-form piece of the Bismut superconnection onto the bundle null $\left(ð_{0}\right)$. The precise statement is given in Theorem 2 . We then establish the surgery isomorphism (2) in the constant rank case and prove that (3), i.e. the additivity of the curvature and of the logarithm of the holonomy under surgery, holds for the perturbed Bismut-Freed connection. The surgery results are stated in detail in Theorem 3. It should be mentioned that the constant rank case is really obtained by reduction to the invertible case, by considering the family $\delta$ acting on weighted Sobolev spaces. It is for this reason that we are able to work with the surgery calculus of [16] throughout and avoid the more delicate logarithmic surgery calculus of [15].

A final comment on our assumption about the boundary family $ð_{0}$. The local anomaly formula can be seen as a consequence of the transgressed form of the family index theorem which follows in turn from the local family index theorem. For an arbitrary spectral section $P$ the index theorem of [18] is not local; as explained above it is first necessary to homotope the family of boundary value problems defined by $ð$ and $P$ to the family of $b$-pseudodifferential operators $\partial+A_{P}$, with invertible boundary family, and then apply the $b$-calculus to the latter. In this operation locality is lost, the index formula only holds at the cohomological level; in particular, unless the boundary family is invertible, or unless it has null spaces of constant dimension in $z \in B$, there is not a transgressed form of the index theorem. This explains our assumption at least as far as the local anomaly formula is concerned. The situation might be slightly better in the case of surgery. First of all it should be possible to extend the isomorphism (2), which is also valid in the constant rank case, to an arbitrary spectral section $P$ obtaining

$$
\operatorname{det}(ð) \leftrightarrow \operatorname{det}_{b}\left(\partial_{\bar{M}^{0}},(\operatorname{Id}-P)\right) \otimes \operatorname{det}_{b}\left(\partial_{\bar{M}^{1}}, P\right)
$$

(see [25] for a special case). Moreover using the results of Müller [20] it is possible to introduce the Bismut-Freed connection on the determinant line bundle $\operatorname{det}_{\mathrm{APS}}(\widehat{\partial}, P)$ defined by the family of generalized Atiyah-Patodi-Singer boundary value problems fixed by $ð$ and $P$. In this context, which is definitely different from the one considered here, one could at least hope for surgery results of the type considered in this paper by employing the techniques of [12].

\section{Section 1. Determinant Line Bundles on Manifolds With Boundary}

Let $\phi: M \rightarrow B$ be a fibration of smooth compact manifolds with fibres diffeomorphic to a fixed even-dimensional manifold with boundary $X$. Let $g_{M / B}$ be a family of exact $b$-metrics and let $E$ be a Hermitian $\mathbb{Z}_{2}$-graded module for the Clifford algebra bundle associated to the vertical $b$-cotangent bundle ${ }^{b} T^{*}(M / B)$. For Clifford algebras we shall use the convention of [17]. Thus for any two covectors $\alpha$ and $\beta$,

$$
\operatorname{cl}(\alpha) \operatorname{cl}(\beta)+\operatorname{cl}(\beta) \operatorname{cl}(\alpha)=2\langle\alpha, \beta\rangle .
$$

Let $\nabla^{E}$ be a vertical (true) connection on $E$ which is assumed to be unitary and Clifford. Let $\delta=\left(ð_{z}\right) \in \operatorname{Diff}_{b, \phi}^{1}(M ; E)$ be the family of Dirac operators associated to these data. We refer the reader to [18 and 19] for some of the notation used in this paper. Let $\mathrm{\partial}_{0}=\left({\delta_{0, z}}_{0}\right) \in \operatorname{Diff}_{\partial \phi}^{1}(\partial M ; E\lceil\partial M)$ be the family of Dirac operators induced on the boundary. By Proposition 1 of [18] (and its Corollary) we can always choose a spectral section $P$ for the boundary family $ð_{0}$. Thus $P$ is 
a smooth family of $0^{\text {th }}$-order pseudodifferential operators, $P \in \Psi_{\partial \phi}^{0}(\partial M ; E\lceil\partial M)$, $P_{z}: L^{2}\left(\partial M_{z} ; E \uparrow \partial M_{z}\right) \rightarrow L^{2}\left(\partial M_{z} ; E \uparrow \partial M_{z}\right)$, which are self-adjoint projection and with the additional property that there exists a positive function $R \in \mathscr{C}^{\infty}(B)$ such that

$$
\mathrm{\partial}_{0, z} u=\lambda u \Rightarrow \begin{cases}P_{z} u=u & \text { if } \lambda>R(z) \\ P_{z} u=0 & \text { if } \lambda<-R(z) .\end{cases}
$$

There are three distinct families of Fredholm operators associated to $\partial$ and a choice of a spectral section $P$ for $ð_{0}$.

First of all we can consider the metric-incomplete setting of Atiyah, Patodi and Singer. For this we need to assume a product metric near the boundary. If $\widehat{M}, \widehat{E}$ denote the total space and the Clifford module in this case, then the smooth family

$$
\left(\widehat{\jmath}^{+}, P\right): \operatorname{Dom}\left(\widehat{\mho}^{+}, P\right) \rightarrow \mathscr{C}^{\infty}\left(\widehat{M} ; \widehat{E}^{-}\right)
$$

with

$$
\operatorname{Dom}\left(\widehat{ठ}^{+}, P\right)=\left\{u \in \mathscr{C}^{\infty}\left(\widehat{M} ; \widehat{E}^{+}\right) ; P(u \uparrow \partial \widehat{M})=0\right\}
$$

is Fredholm, when considered as a family acting on the appropriate Sobolev completions, with null space consisting of $\mathscr{C}^{\infty}$ functions. According to Quillen [23] (see also [10]) there is a well defined determinant line bundle associated to $\left(\widehat{\partial}^{+}, P\right)$. We

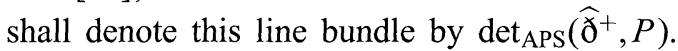

If we add a cylindrical end $(-\infty, 0]_{v} \times \partial \widehat{M}$ to $\widehat{M}$ and we make the usual change of variable $x=\exp (v)$, then we obtain a fibration of $b$-manifolds which are product type near the boundary. More generally if the metrics on the fibres are exact we can define, following [18], two distinct families of Fredholm operators. The first one is a (local) family of Fredholm operators acting on Hilbert spaces which are finite dimensional extensions of weighted Sobolev spaces. We denote the associated determinant line bundle by $\operatorname{det}_{b}(ð, P)$. The second family, in this complete setting, is obtained by perturbing $\varnothing$ by an element $A_{P} \in \Psi_{b, \phi}^{-\infty}(M, E)$, i.e. a smooth family of $b$-pseudodifferential operators of order $(-\infty)$, so that the boundary operators of the perturbed family are all invertible; acting on unweighted Sobolev spaces the perturbed family $ð+A_{P} \in \Psi_{b, \phi}^{1}(M, E)$ is Fredholm. Thus there exists a well defined determinant bundle which we will simply denote by $\operatorname{det}_{b}\left(ð+A_{P}\right)$. Since, as explained in Proposition 6 of [18], these two families are homotopic it follows that

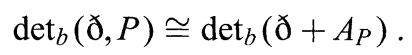

In the product case we can use Lemma 2 in [18] obtaining a natural isomorphism

$$
\operatorname{det}_{\mathrm{APS}}(\widehat{ð}, P) \cong \operatorname{det}_{b}(ð, P) \text {. }
$$

Directly from the family index theorem proved in [18] we obtain (see [1]) the following result.

Theorem 1. For the Chern class of the determinant line bundle $\operatorname{det}_{b}(\partial, P)$ the following formula holds:

$$
c_{1}\left(\operatorname{det}_{b}(ð, P)\right)=\left[\frac{1}{(2 \pi i)^{\frac{n}{2}}} \int_{M / B} \widehat{A}(M / B) \mathrm{Ch}^{\prime}(E)-\frac{1}{2} \widehat{\eta}_{P}\right]_{[2]} \quad \text { in } H^{2}(B) .
$$

If the fibre metrics are of product type near the boundary then

$$
c_{1}\left(\operatorname{det}_{\mathrm{APS}}(\widehat{ð}, P)\right)=c_{1}\left(\operatorname{det}_{b}(ð, P)\right) .
$$


It should be remarked at this point that if $Q$ is a different spectral section for $\mathrm{\delta}_{0}$, then the relative index theorem of [18] gives a way to relate the Chern classes of $\operatorname{det}_{b}(ð, P)$ and $\operatorname{det}_{b}(ð, Q)$ :

$$
c_{1}\left(\operatorname{det}_{b}(ð, Q)\right)=c_{1}\left(\operatorname{det}_{b}(ð, P)\right)+\operatorname{Ch}([P-Q])_{[2]} .
$$

It is proved in [19] that the formal differences of spectral sections associated to a Dirac operator exhaust all of $K^{0}(B)$ (see Proposition 11). Since the Chern character gives an isomorphism $\mathrm{Ch}: K^{0}(B) \otimes \mathbb{C} \rightarrow H^{\mathrm{ev}}(B)$ we obtain the following somewhat surprising result:

Proposition 1. There always exists a spectral section $Q$ such that

$$
c_{1}\left(\operatorname{det}_{b}(ð, Q)\right)=0 .
$$

Theorem 1 gives a way of measuring the non-triviality of the determinant line bundle associated to a family of Dirac operators and the choice of a spectral section $P$ for the induced boundary family. As explained in the introduction it is however important to investigate the Hermitian geometry of $\operatorname{det}_{\mathrm{APS}}(\widehat{ð}, P)$ and $\operatorname{det}_{b}(ð, P)$, as in $[23,10]$. In order to carry out this program we need to impose some restrictions on the boundary family $ð_{0}=\left(\partial_{0, z}\right) \in \operatorname{Diff}_{\partial \phi}^{1}(\partial M ; E \uparrow \partial M)$. The assumption we need to make is the following:

The null spaces of the boundary family ${\mathrm{\partial}_{0, z}}_{2}, z \in B$, are of constant dimension .

We refer to assumption (1.5) as the constant rank case. Under this assumption we can and we shall choose the spectral section $P$ to be $\Pi_{\geqq}$, with $\Pi_{\geqq}^{z}$ equal to the spectral projection corresponding to the non-negative eigenvalues of the boundary operators $ð_{0, z}$.

\section{Section 2. b-Zeta Functions and $b$-Quillen Metrics}

In order to investigate the geometry of the determinant line bundles $\operatorname{det}_{\mathrm{APS}}\left(\widehat{\varnothing}, \Pi_{\geqq}\right)$ and $\operatorname{det}_{b}\left(ð, \Pi_{\geqq}\right)$we first assume the boundary operators to be invertible. Thus we assume

$$
\text { For each } z \in B \text { the boundary operator } ð_{0, z} \text { is invertible . }
$$

We shall treat the constant rank case in Sect. 8.

Consider the determinant line bundle $\operatorname{det}_{b}\left(ð, \Pi_{\geqq}\right)$. This is simply the determinant line bundle associated to the family of Fredholm operators

$$
\mathrm{\partial}_{z}^{+}: H_{b}^{1}\left(M_{z} ; E_{z}^{+}\right) \rightarrow L_{b}^{2}\left(M_{z} ; E_{z}^{-}\right) .
$$

Assumption (2.1) implies that $0 \notin \operatorname{spec}\left({\varlimsup_{0, z}}^{2}\right.$ for each $z \in B$. Thus the $L_{b}^{2}$-spectrum of $\delta_{z}^{2}$ is discrete near zero; following [23] and [10] we can then give the following description of $\operatorname{det}_{b}\left(ð, \Pi_{\geqq}\right)$.

There exists a $\delta>0$ such that

$$
\left.\operatorname{spec}\left({\chi_{0, z}^{2}}^{2}\right) \cap w \in \mathbb{R}:|w| \leqq \delta\right\}=\emptyset
$$

for each $z \in B$. Thus (see [17]) the $L_{b}^{2}$-spectrum of the self-adjoint operator $\partial_{z}^{2}$ is discrete with finite multiplicity precisely in the region $S(\delta)=\{x \in \mathbb{R}: 0 \leqq x \leqq \delta\}$. 
Let $\lambda \in S(\delta)$ and consider the open subset of $B$,

$$
U_{\lambda}=\left\{z \in B: \lambda \notin \operatorname{spec}\left({ }_{z}^{2}\right)\right\} .
$$

Since the spectrum is discrete in the region $S(\delta)$ it is clear that the $U_{\lambda}$ 's give an open covering $\mathscr{U}$ of the manifold $B$. We denote by $P_{\lambda}=\left(P_{\lambda}^{z}\right)$ the family of spectral projections corresponding to the interval $[0, \lambda) \subset \mathbb{R}$, i.e. the orthogonal projections, in $L_{b}^{2}\left(M_{z} ; E\right)$, onto the direct sum of the eigenvalue which are less than $\lambda$. Since $\delta^{2}$ is $\mathbb{Z}_{2}$-graded even we see that $P_{\lambda}^{z}$ is also $\mathbb{Z}_{2}$-graded even. Following the notation in [2] we consider the $\mathbb{Z}_{2}$-graded complex vector bundle $H_{\lambda}$ on $U_{\lambda}$ defined by the range of the family $P_{\lambda}$ :

$$
\left(H_{\lambda}\right)_{z}=\left(H_{\lambda}^{+}\right)_{z} \oplus\left(H_{\lambda}^{-}\right)_{z}=\left(\operatorname{ran} P_{\lambda}^{z,+}\right) \oplus\left(\operatorname{ran} P_{\lambda}^{z,-}\right) .
$$

The same arguments given in [18] for the null bundle of the family $\partial$ show that $H_{\lambda}$ is a smooth vector bundle on $U_{\lambda}$. We consider the line bundle over $U_{\lambda}$,

$$
\operatorname{det}\left(H_{\lambda}\right)=\left(\Lambda^{\mathrm{max}} H_{\lambda}^{+}\right)^{*} \otimes\left(\Lambda^{\mathrm{max}} H_{\lambda}^{-}\right) .
$$

If $\mu \in S(\delta)$ and $\lambda<\mu$ then on $U_{\lambda} \cap U_{\mu}$ we certainly have

$$
H_{\mu}=H_{\lambda} \oplus H_{[\lambda, \mu)},
$$

with $H_{[\lambda, \mu)}$ equal to the direct sum of the eigenspaces corresponding to the eigenvalues in the interval $[\lambda, \mu)$. Thus

$$
\operatorname{det}\left(H_{\mu}\right)=\operatorname{det}\left(H_{\lambda}\right) \otimes \operatorname{det}\left(H_{[\lambda, \mu)}\right) .
$$

We can define a line bundle by gluing $\operatorname{det}\left(H_{\lambda}\right)$ and $\operatorname{det}\left(H_{\mu}\right)$ on $U_{\lambda} \cap U_{\mu}$ through the non-zero function $\operatorname{det}\left({\varlimsup_{(\lambda, \mu)}^{+}}_{(\lambda)}\right.$ induced by the isomorphism

$$
{\chi_{(\lambda, \mu)}^{+}}^{+} ð^{+} \uparrow H_{[\lambda, \mu)}^{+}: H_{[\lambda, \mu)}^{+} \leftrightarrow H_{[\lambda, \mu)}^{-} .
$$

We denote this line bundle by $\operatorname{det}_{b}(ð)$. As explained in [10] the two line bundles $\operatorname{det}_{b}\left(ð, \Pi_{\geqq}\right)$and $\operatorname{det}_{b}(ð)$ are canonically identified; we shall often think of $\operatorname{det}_{b}\left(ð, \Pi_{\geqq}\right)$and $\operatorname{det}_{b}(ð)$ as the same object.

For families of Dirac operators acting on closed manifolds, Quillen has introduced a natural metric on the determinant bundle. This is nowadays referred to as the Quillen metric; it is defined through the derivative at the origin of the zeta function of the positive Dirac Laplacians.

Let $A \in \Psi_{b}^{*}(X ; E)$ be a positive elliptic $b$-pseudodifferential operator acting between the smooth sections of a vector bundle $E$ on a manifold with boundary $X$. In [22] the complex powers $A^{-s}, \operatorname{Re} s \gg 0$, were studied. These complex powers are not trace class in general and one must take a regularization of the trace functional, the $b$-trace [17], in order to define the $b$-zeta function of $A$. Thus for $\operatorname{Re} s$ large,

$$
b_{\zeta}(A, s) \stackrel{\text { def }}{=} b-\operatorname{Tr}\left(A^{-s}\right) .
$$

The reader is referred to $[22,17]$ for the proofs of the main properties of $b \zeta$ that will be used in the sequel. In our particular case it follows that for each $z \in U_{\lambda}$,

$$
{ }_{\zeta} \zeta\left(s, \mathrm{\partial}_{z}^{-} \mathrm{\circlearrowright}_{z}^{+}, \lambda\right)=b-\operatorname{Tr}\left(P_{(\lambda, \infty)}^{z,+}\left(\varlimsup_{z}^{-} \mathrm{ठ}_{z}^{+}\right)^{-s}\right)
$$


is well defined for $\operatorname{Re} s \gg 0$. Here $\lambda<\delta$ and $P_{[\lambda, \infty)}^{z,+}=\operatorname{Id}-P_{\lambda}^{z,+}$. It can be proved, either by studying the structure of the Schwartz kernel of the complex powers of $A$ [22] or by using the Mellin transform and the asymptotic of the heat kernel for small times [17], that the complex function ${ }^{b} \zeta\left(s, \partial_{z}^{-} \chi_{z}^{+}, \lambda\right)$ admits a meromorphic extension to the whole complex plane. The point $s=0$, an a priori pole, is regular. Thus ${ }^{b} \zeta^{\prime}\left(0, \partial_{z}^{-} \chi_{z}^{+}, \lambda\right)$ is well defined for each $z \in U_{\lambda}$. Since $ð \in \operatorname{Diff}_{b, \phi}^{1}(M ; E)$ is a smooth family of operators, we see that, as $z$ varies in $U_{\lambda},{ }^{b} \zeta^{\prime}\left(0, \partial_{z}^{-} \partial_{z}^{+}, \lambda\right)$ defines a smooth function on $U_{\lambda}$. We denote this function by ${ }^{b} \zeta^{\prime}\left(0, \partial^{-} \partial^{+}, \lambda\right)$. It is important to note that if $z \in U_{\lambda} \cap U_{\mu}$, with $\lambda<\mu<\delta$, then for $\operatorname{Re} s$ large,

$$
{ }^{b} \zeta\left(s, \partial_{z}^{-} \chi_{z}^{+}, \lambda\right)-{ }^{b} \zeta\left(s, \partial_{z}^{-} \chi_{z}^{+}, \mu\right)=b-\operatorname{Tr}\left(P_{(\lambda, \mu]}^{z,+}\left(\chi_{z}^{-} \chi_{z}^{+}\right)^{-s}\right) .
$$

Since $b-\operatorname{Tr}=\operatorname{Tr}$ on trace class operators it follows that

$$
{ }^{b} \zeta\left(s, \partial_{z}^{-} \chi_{z}^{+}, \lambda\right)={ }^{b} \zeta\left(s, \partial_{z}^{-} \chi_{z}^{+}, \mu\right)+\sum_{i=1}^{m} \lambda_{i}^{-s} \operatorname{Re} s \gg 0
$$

with $0<\lambda<\lambda_{1} \leqq \cdots \leqq \lambda_{m} \leqq \mu<\delta$ an enumeration of the eigenvalues of $\partial_{z}^{-} \partial_{z}^{+}$ between $\lambda$ and $\mu$. This implies directly, by meromorphic continuation, the key formula

$$
{ }^{b} \zeta^{\prime}\left(0, \partial_{z}^{-} \chi_{z}^{+}, \lambda\right)={ }^{b} \zeta^{\prime}\left(0, \partial_{z}^{-} \partial_{z}^{+}, \mu\right)-\sum_{i=1}^{m} \log \lambda_{i} .
$$

To define the Quillen metric we now proceed as in [23]: the $L_{b}^{2}$-metrics associated to the family of exact $b$-metrics $g_{M / B}$ induce a metric on $H_{[0, \lambda)}$ and thus a metric $|\cdot|_{\lambda}$ on $\left(\operatorname{det}_{b}(ð)\right) \mid U_{\lambda}=\operatorname{det}\left(H_{[0, \lambda)}\right)$. We define the Quillen metric as

$$
\|\cdot\|_{b, Q}=e^{-b^{\zeta^{\prime}\left(0, \gamma^{-} \gamma^{+}, \lambda\right) / 2}|\cdot|_{\lambda} .}
$$

Formula (2.4) implies that $\|\cdot\|_{b, Q}$ is globally defined on $\operatorname{det}_{b}\left(\partial, \Pi_{\geqq}\right)$.

We end this section by computing the variation of the function ${ }^{b} \zeta^{\prime}\left(0, \partial_{z}^{-} \partial_{z}^{+}, \lambda\right)$. We will denote by $\partial / \partial z$ a generic partial derivative in a fixed coordinate patch contained in $U_{\lambda}$.

Lemma 1. Over the set $U_{\lambda}$ the following formula holds

$$
\frac{\partial}{\partial z}\left(b_{\zeta} \zeta\left(s, \partial_{z}^{-} \chi_{z}^{+}, \lambda\right)\right)=-s\left(b-\operatorname{Tr}\left(P_{(\lambda, \infty)}^{+, z}\left(\frac{\partial}{\partial z}\left(\partial_{z}^{-} \partial_{z}^{+}\right)\right)\left(\partial_{z}^{-} ठ_{z}^{+}\right)^{-s-1}\right)\right) .
$$

Proof. For Re $s$ large the $b$-zeta function can be expressed, using the Mellin transform, in terms of the $b$-trace of the heat kernel:

$$
{ }^{b} \zeta\left(s, \partial_{z}^{-} \delta_{z}^{+}, \lambda\right)=\mathrm{M}\left[b-\operatorname{Tr}\left(P_{(\lambda, \infty)}^{z,+} e^{-t \delta_{z}^{-} \delta_{z}^{+}}\right)\right](s)=\frac{1}{\Gamma(s)} \int_{0}^{\infty} b-\operatorname{Tr}\left(P_{(\lambda, \infty)}^{z,+} e^{-t \delta_{z}^{-} \delta_{z}^{+}}\right) t^{s-1} d t,
$$

where $\mathrm{M}$ denotes the Mellin transform. The derivative with respect to $z$ of the last term above is the sum of two terms; one involving the derivative of the projection and the other involving the derivative of the heat kernel. The first contribution vanishes and we are left with the task of proving that

$$
b-\operatorname{Tr}\left(P_{(\lambda, \infty)}^{z,+} \frac{\partial}{\partial z} e^{-t \delta_{z}^{-} \gamma_{z}^{+}}\right)=-t\left(b-\operatorname{Tr}\left(P_{(\lambda, \infty)}^{z,+}\left(\frac{\partial}{\partial z}{\delta_{z}^{-}}_{z}^{-} \gamma_{z}^{+}\right) e^{-t \delta_{z}^{-} \delta_{z}^{+}}\right)\right) .
$$


We apply Duhamel's principle to compute the derivative of the heat kernal obtaining, for the left-hand side, the integral

$$
-b-\operatorname{Tr}\left(\int_{0}^{t} e^{-(t-s) \delta_{z}^{-} \partial_{z}^{+}} P_{(\lambda, \infty)}^{z,+}\left(\frac{\partial}{\partial z} \partial_{z}^{-} \partial_{z}^{+}\right) e^{-s \partial_{z}^{-} \partial_{z}^{+}} d s\right) .
$$

Here we used the fact that $\left[\exp \left(-t{\delta_{z}^{-}}_{z}^{-} \partial_{z}^{+}\right), P_{(\lambda, \infty)}^{z,+}\right]=0$. We rewrite this term as the sum of the right-hand side of (2.7) and a term which involves the $b$-trace of a commutator:

$$
\begin{aligned}
& -b-\operatorname{Tr}\left(P_{(\lambda, \infty)}^{z,+} t\left(\frac{\partial}{\partial z} \delta_{z}^{-}{\delta_{z}^{+}}^{+}\right) e^{-t \delta_{z}^{-} \partial_{z}^{+}}\right) \\
& -b-\operatorname{Tr}\left(\int_{0}^{t}\left[e^{-(t-s) \delta_{z}^{-} \partial_{z}^{+}}, P_{(\lambda, \infty)}^{z,+}\left(\frac{\partial}{\partial z} \delta_{z}^{-} \partial_{z}^{+}\right) e^{-s \delta_{z}^{-} \delta_{z}^{+}}\right] d s\right) \text {. }
\end{aligned}
$$

Applying the $b$-trace identity [17] to the latter term we obtain

$$
\begin{aligned}
& b-\operatorname{Tr}\left(P_{(\lambda, \infty)}^{z,+} \frac{\partial}{\partial z} e^{-t \delta_{z}^{-} \partial_{z}^{+}}\right)=-b-\operatorname{Tr}\left(P_{(\lambda, \infty)}^{z,+} t\left(\frac{\partial}{\partial z} \partial_{z}^{-} \partial_{z}^{+}\right) e^{-t \delta_{z}^{-} \partial_{z}^{+}}\right) \\
& \quad+\frac{\operatorname{Tr}}{2 \pi i}\left(\int_{\mathbb{R}} \int_{0}^{t} \frac{d}{d w}\left(e^{-(t-s)\left(w^{2}+\partial_{0, z}^{2}\right)}\right) I\left(P_{(\lambda, \infty)}^{z,+}\left(\frac{\partial}{\partial z} \partial_{z}^{-} \partial_{z}^{+}\right) e^{-s \delta_{z}^{-} \partial_{z}^{+}}, w\right) d s d w\right),
\end{aligned}
$$

where following the identifications in [18] we have used the identity $I\left(\partial_{z}^{-} \partial_{z}^{+}, w\right)=$ $w^{2}+\left(ð_{0, z}\right)^{2}$. Notice that this also implies that

$$
I\left(\frac{\partial}{\partial z} \partial_{z}^{-} ð_{z}^{+}, w\right)=\frac{\partial}{\partial z}\left(ð_{0, z}\right)^{2} .
$$

Recall that $\lambda<\delta$ with $\delta$ as in (1.2). Thus the same analysis given in [17] for the projection operator onto the null space of an elliptic $b$-differential operator shows that the image under the blow down map of the Schwartz kernel of $P_{\lambda}^{z,+}$ is given by a continuous section of the external tensor product $E \bowtie E^{*}$ over $M_{z} \times M_{z}$ which is smooth in the interior and vanishes over the boundary. Thus $I\left(P_{\lambda}^{z,+}, w\right)=0$ for each $w \in \mathbb{C}$ so that

$$
I\left(P_{(\lambda, \infty)}^{z,+}, w\right)=I\left(\operatorname{Id}-P_{\lambda}^{z,+}, w\right)=\operatorname{Id} .
$$

Thus we can rewrite the last integral in the right-hand side of (2.8) as

$$
\frac{1}{2 \pi i}\left(\int_{0}^{t}(t-s) \operatorname{Tr}\left(\frac{\partial}{\partial z}{\partial_{0, z}}\right) e^{-t\left(\partial_{0, z}\right)^{2}} d s\right)\left(\int_{\mathbb{R}}(-2 w) e^{-w^{2} t} d w\right),
$$

which is clearly zero. Thus (2.7) holds and the proof of the lemma is complete.

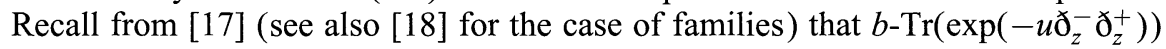
has an asymptotic expansion for small $u$. It is obvious that an analogous asymptotic expansion holds for $b-\operatorname{Tr}\left(P_{(\lambda, \infty)}^{z,+} \exp \left(-u \mathrm{\delta}_{z}^{-} \mathrm{\partial}_{z}^{+}\right)\right)$:

$$
b-\operatorname{Tr}\left(P_{(\lambda, \infty)}^{z,+} e^{-u ð_{z}^{-} \delta_{z}^{+}}\right) \sim \sum_{k=-\frac{n}{2}}^{\infty} a_{k}(z) u^{k}
$$


with $n$ equal to the dimension of the fibre $M_{z}$. Formula (2.7) implies an asymptotic expansion for $b-\operatorname{Tr}\left(P_{(\lambda, \infty)}^{z,+}\left(\frac{\partial}{\partial z} \delta_{z}^{-} \delta_{z}^{+}\right) \exp \left(-u ð_{z}^{-} \delta_{z}^{+}\right)\right)$:

$$
b-\operatorname{Tr}\left(P_{(\lambda, \infty)}^{z,+}\left(\frac{\partial}{\partial z} \partial_{z}^{-} \partial_{z}^{+}\right) e^{-u \delta_{z}^{-} \partial_{z}^{+}}\right) \sim-\sum_{k=-\frac{n}{2}}^{\infty} \frac{\partial}{\partial z} a_{k}(z) u^{k},
$$

which in turn gives an asymptotic expansion for $b-\operatorname{Tr}\left(P_{(\lambda, \infty)}^{z,+}\left(\frac{\partial}{\partial z} \partial_{z}^{-} \partial_{z}^{+}\right)\left(\partial_{z}^{-} \partial_{z}^{+}\right)^{-1}\right.$ $\exp \left(-t{\delta_{z}}_{z}^{-}{\partial_{z}^{+}}^{+}\right)$, simply obtained by taking the integral from $t$ to $\infty$ of formula (2.9). If we denote by

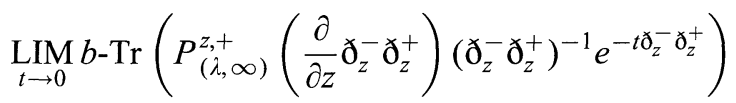

the coefficient of $t^{0}$ in this asymptotic expansion, then we obtain from Lemma 6 , as in [2], the following important result

Proposition 2. On the open set $U_{\lambda}$ we have

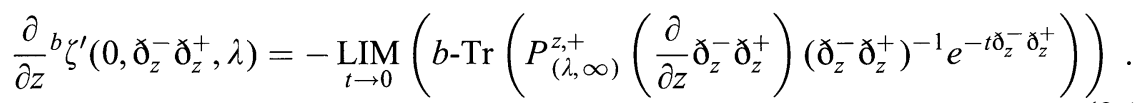

\section{Section 3. The Bismut-Freed Connection in the b-Context}

In this section we introduce a natural connection ${ }^{b} \nabla^{\text {det }}$ compatible with the $b$ Quillen metric. Let $\lambda<\delta$, with $\delta$ as in (1.2). Observe incidentally that over the set $U_{\lambda} \subset B$ the operator $\partial_{(\lambda, \infty)}=P_{(\lambda, \infty)}$ ठ has $H_{[0, \lambda)}$ as its kernel bundle. As in [2] we consider the superconnection $\widehat{\mathbb{A}}_{\lambda}=\partial_{(\lambda, \infty)}+\mathbb{A}_{[1]}$ with $\mathbb{A}_{[1]}$ the one-form piece of the Bismut superconnection. Let $\widehat{\mathbb{A}}_{\lambda, t}=t^{\frac{1}{2}} \mathrm{\partial}_{(\lambda, \infty)}+\mathbb{A}_{[1]}$ be the rescaled superconnection associated to $\widehat{\mathbb{A}}_{\lambda}$. Define two differential 1 -forms $\alpha_{b}^{ \pm}(t, \lambda)_{[1]} \in \mathscr{C}^{\infty}\left(U_{\lambda}, \Lambda^{1}\right)$ by

$$
\alpha_{b}^{ \pm}(t, \lambda)_{[1]}=\left(b-\operatorname{Tr}_{ \pm}\left(\frac{\partial \widehat{\mathbb{A}}_{\lambda, t}}{\partial t} e^{-\left(\widehat{\mathbb{A}}_{\lambda, t}\right)^{2}}\right)\right)_{[1]} \text {. }
$$

More explicitly

$$
\begin{aligned}
\alpha_{b}^{ \pm}(t, \lambda)_{[1]} & =-\frac{1}{2} b-\operatorname{Tr}_{ \pm}\left(\partial_{(\lambda, \infty)}^{\mp}\left[\mathbb{A}_{[1]}, \partial_{(\lambda, \infty)}^{ \pm}\right] e^{-t\left(\partial_{(\lambda, \infty)}\right)^{2}}\right) \\
& =-\frac{1}{2} b-\operatorname{Tr}_{ \pm}\left(P_{(\lambda, \infty)} \partial^{\mp}\left[\mathbb{A}_{[1]}, \partial^{ \pm}\right] e^{-t\left(\partial^{2}\right.}\right) .
\end{aligned}
$$

It follows from [17] (Proposition (7.27)) and [18] (Proposition 20) that $\alpha_{b}^{ \pm}(t, \lambda)_{[1]}$ has an asymptotic expansion for $t$ small:

$$
\alpha_{b}^{ \pm}(t, \lambda)_{[1]} \sim \sum_{k=-\frac{n}{2}}^{\infty} t^{k / 2} a_{k}^{ \pm}
$$

Thus

$$
\beta_{b}^{ \pm}(\lambda)=2 \underset{s \rightarrow 0}{\operatorname{LIM}} \int_{s}^{\infty} \alpha_{b}^{ \pm}(t, \lambda)_{[1]}
$$

is well defined. The 1-form piece of the Bismut superconnection induces, through the spectral projection $P_{[0, \lambda)}$, a $\mathbb{Z}_{2}$-graded connection on $H_{[0, \lambda)}$ which in turn induces 
a connection ${ }^{b} \nabla^{\lambda}$ on $\left(\operatorname{det}_{b} \partial\right) \uparrow U_{\lambda}$. This connection is compatible with the metric $|\cdot|_{\lambda}$ induced by the $L_{b}^{2}$-inner product. To define a connection compatible with the Quillen metric we consider on $\left(\operatorname{det}_{b} \partial\right) \uparrow U_{\lambda}$ the connection

$$
{ }^{b} \nabla^{\operatorname{det}}={ }^{b} \nabla^{\hat{\lambda}}+\beta_{b}^{+}(\lambda) \text {. }
$$

One proves as in the boundaryless case that ${ }^{b} \nabla^{\lambda}+\beta_{b}^{+}(\lambda)$ and ${ }^{b} \nabla^{\mu}+\beta_{b}^{+}(\mu)$ agree over the set $U_{\lambda} \cap U_{\mu}$ once we identify $\operatorname{det}\left(H_{[0, \lambda)}\right)$ and $\operatorname{det}\left(H_{[0, \mu)}\right)$ by multiplying by the non-vanishing section $\operatorname{det}\left(\mathrm{\delta}_{(\lambda, \mu)}^{+}\right)$of $\operatorname{det}\left(H_{[\lambda, \mu)}\right)$. Thus ${ }^{b} \nabla^{\operatorname{det}}$ is globally defined on $\operatorname{det}_{b}\left(ð, \Pi_{\geqq}\right)$. To prove that it is compatible with the Quillen metric we need the following

Lemma 2. Over the set $U_{\lambda}$

$$
\begin{aligned}
\alpha_{b}^{-}(t, \lambda)_{[1]} & =\overline{\alpha_{b}^{+}(t, \lambda)_{[1]}}, \\
d\left({ }^{b} \zeta^{\prime}\left(0, \partial^{-} \chi^{+}, \lambda\right)\right) & =-\left(\beta_{b}^{+}(\lambda)+\beta_{b}^{-}(\lambda)\right) .
\end{aligned}
$$

Proof. From the expression (3.2) it follows that $\overline{\alpha_{b}^{+}(t, \lambda)_{[1]}}$ is equal to the complex conjugate of

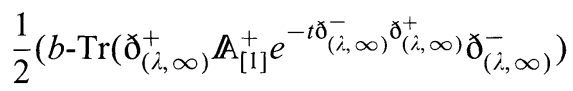

$$
\begin{aligned}
& -b-\operatorname{Tr}\left(\mathbb{A}_{[1]}^{-} \mathrm{\partial}_{(\lambda, \infty)}^{+} e^{\left.\left.-t \mathrm{\delta}_{(\lambda, \infty)}^{-} \widehat{\partial}_{(\lambda, \infty)}^{+} \mathrm{\partial}_{(\lambda, \infty)}^{-}\right)\right)}\right. \\
& +\frac{1}{2}\left(b-\operatorname{Tr}\left[\mathrm{\partial}_{(\lambda, \infty)}^{-}, \mathrm{\partial}_{(\lambda, \infty)}^{+} \mathbb{A}_{[1]}^{+} e^{-t \partial_{(\hat{\lambda}, \infty)}^{-} \partial_{(\lambda, \infty)}^{+}}\right]\right. \\
& -b-\operatorname{Tr}\left[\partial_{(\lambda, \infty)}^{-}, \mathbb{A}_{[1]} \partial_{(\lambda, \infty)}^{+} e^{\left.\left.-t \delta_{(\lambda, \infty)}^{-} \partial_{(\lambda, \infty)}^{+}\right]\right) .}\right.
\end{aligned}
$$

Locally in $U \subset U_{\lambda}$ we can write $\mathbb{A}_{[1]}^{ \pm}=d+\omega^{ \pm}$with $\omega^{ \pm} \in \mathscr{C}^{\infty}\left(\phi^{-1}\left(U, \phi^{*} \Lambda^{1} B \otimes\right.\right.$ hom $(E)))$. With the identifications explained in [18] we have

$$
I\left(\partial_{(\lambda, \infty)}^{ \pm}, w\right)=I\left(\partial^{ \pm}, w\right)= \pm i w+ð_{0} \quad w \in \mathbb{C}
$$

since, as already remarked, $I\left(P_{[0, \lambda)}, w\right)=0$. Recall also that

$$
\omega^{+} \uparrow \partial M=\omega^{-} \uparrow \partial M .
$$

The last two lines in (3.6) are equal to

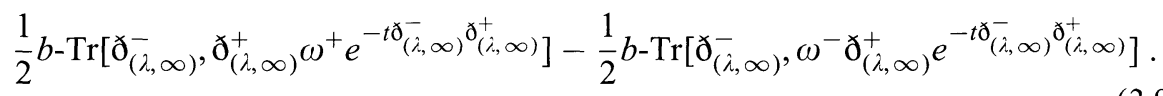

Application of the $b$-trace identity ([17]) gives the equality of (3.9) with

$$
\begin{aligned}
\int_{\mathbb{R}} & \left(\frac{\operatorname{Tr}}{4 \pi}\left(i w+\succsim_{0}\right)\left(\omega^{+} \uparrow \partial M\right) e^{-t\left(w^{2}+\partial_{0}^{2}\right)}\right) d w \\
- & \int_{\mathbb{R}}\left(\frac{\operatorname{Tr}}{4 \pi}\left(\omega^{-} \uparrow \partial M\right)\left(i w+\succsim_{0}\right) e^{-t\left(w^{2}+\partial_{0}^{2}\right)}\right),
\end{aligned}
$$


which is easily seen to be zero. Next we consider the complex conjugate of the first two lines in (3.6). Since $\mathbb{A}_{[1]}$ is compatible with the $L_{b}^{2}$-metrics defined by $g_{M / B}$ we find

$$
\begin{aligned}
& \overline{\alpha_{b}^{+}(t, \lambda)_{[1]}}=\frac{1}{2} b-\operatorname{Tr}_{-}\left(e^{-t{\delta_{(i, \infty)}^{+}}_{(\lambda, \infty)}^{\partial_{(\lambda,}^{-}} \partial_{(\lambda, \infty)}^{+}\left(-\mathbb{A}_{[1]}^{+}\right) \partial_{(\lambda, \infty)}^{-}}\right)
\end{aligned}
$$

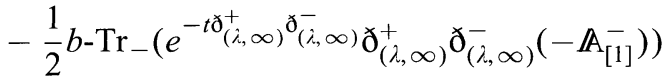

$$
\begin{aligned}
& =\alpha_{b}^{-}(t, \lambda)_{[1]}+\frac{1}{2} b-\operatorname{Tr}\left[{\chi_{(\lambda, \infty)}^{+}}^{+} \mathbb{A}_{[1]}^{+} \chi_{(\lambda, \infty)}^{-}, e^{\left.-t \delta_{(\lambda, \infty)}^{+} \partial_{(\lambda, \infty)}^{-}\right]}\right. \\
& -\frac{1}{2} b-\operatorname{Tr}\left[\mathrm{\partial}_{(\lambda, \infty)}^{+} \mathrm{\partial}_{(\lambda, \infty)}^{-} \mathbb{A}_{[1]}^{-}, e^{\left.-t{\mathrm{\partial}_{(\lambda, \infty)}^{+}}_{(\lambda, \infty)}^{-}\right]}\right. \\
& =\alpha_{b}^{-}(t, \lambda)_{[1]},
\end{aligned}
$$

the last step being obtained by applying once again the $b$-trace identity to the terms involving commutators together with (3.8).

To establish the second formula in (3.6) we write

$$
\beta_{b}^{+}(\lambda)+\beta_{b}^{-}(\lambda)=\operatorname{LIM}_{t \rightarrow 0} b-\operatorname{Tr}_{+}\left(P_{(\lambda, \infty)}^{+}\left[\mathbb{A}_{[1]}, \partial^{-} \partial^{+}\right]\left(\partial^{-} \partial^{+}\right)^{-1} e^{-t \partial^{-} \partial^{+}}\right) .
$$

Using the fact that $P_{(\lambda, \infty)}^{+}$is a projection commuting with $\partial^{-\partial^{+}}$we obtain

$$
\begin{aligned}
& \beta_{b}^{+}(\lambda)+\beta_{b}^{-}(\lambda)=\operatorname{LIM}_{t \rightarrow 0} b-\operatorname{Tr}_{+}\left(P_{(\lambda, \infty)}^{+}\left(d \partial^{-} \partial^{+}\right)\left(\partial^{-} \partial^{+}\right)^{-1} e^{-t \partial^{-} \partial^{+}}\right) \\
& -\operatorname{LIM}_{t \rightarrow 0} b-\operatorname{Tr}_{+}\left[P_{(\lambda, \infty)}^{+} \partial^{-} \partial^{+}, P_{(\lambda, \infty)}^{+} \omega^{+}\left(\partial^{-} \partial^{+}\right)^{-1} e^{-t \partial^{-} \partial^{+}}\right] .
\end{aligned}
$$

The $b$-trace of the commutator vanishes since the $w$-derivative of the indicial family of the first term in the commutator is odd in $w$ whereas the indicial family of the second term is even. On the other hand by Proposition 2 the first term on the right-hand side is precisely $-d^{b} \zeta^{\prime}\left(0, \partial^{-} \chi^{+}, \lambda\right)$ proving the lemma.

Proceeding as in [2] we obtain from Lemma 2 the following.

Proposition 3. The connection ${ }^{b} \nabla^{\mathrm{det}}$ is compatible with the $b$-Quillen metric $\|\cdot\|_{b, Q}$ on $\operatorname{det}_{b}\left(\partial, \Pi_{\geqq}\right)$.

\section{Section 4. Surgery}

Let $\psi: M \rightarrow B$ be a fibration of closed Riemannian even dimensional manifolds with fibre diffeomorphic to a fixed compact closed manifold $X$ and let $E$ be a vertical Hermitian Clifford module as in [2]. We denote by $g_{M / B}$ the metric on the vertical tangent bundle $T(M / B)$; thus for each $z \in B M_{z}=\psi^{-1}(z)$ is endowed with the metric $g_{z}=g_{M / B} \uparrow T M_{z}$. Let $ð$ be the associated family of Dirac operators. Let $H$ be a codimension one embedded submanifold of $M$. We assume that $H$ fibres over $B$; thus there exists a codimension one embedded submanifold $Y$ of $X$ and a fibre bundle $\chi: H \rightarrow B$ with fibres diffeomorphic to $Y$.

We assume, for simplicity, that $H$ separates $M$; thus $\psi: M \rightarrow B$ is the union of two fibrations $\psi_{i}: M^{i} \rightarrow B, i=0,1$ with common boundary equal to $H: M_{z}=$ $M_{z}^{0} \bigcup_{H_{z}} M_{z}^{1}$ for each $z \in B$. 
Let $x \in \mathscr{C}^{\infty}(M)$ be a defining function for $H$ (thus $H=\{x=0\}$ and $d x \neq 0$ on $H$ ). It should be remarked that one of the two fibrations with boundary, say $M^{0}$, will have the normal vector field to its boundary, $\partial / \partial x$, oriented in the outward direction.

We consider the fibration $\bar{M}=\bar{M}^{0} \sqcup \bar{M}^{1}$ obtained by taking the disjoint union of the compactification $\bar{M}^{i}, i=0,1$ of the fibrations obtained by attaching a cylindrical end to $M_{i}$.

Consider the family of Riemannian metrics

$$
g_{M / B}(\varepsilon)=\frac{|d x|^{2}}{x^{2}+\varepsilon^{2}}+g_{M / B} .
$$

We shall also use the shorter notation $g_{z}(\varepsilon)$ for the metric (4.1) restricted to the fibre $M_{z}$.

The limit metric $g_{M / B}(0) \equiv g(0)$ endows the fibration $\bar{\psi}: \bar{M}=\bar{M}^{0} \sqcup \bar{M}^{1} \rightarrow B$ with a vertical family of exact $b$-metrics. Equivalently $g_{z}(0)$ defines on $M_{z} \backslash H_{z}$ the structure of a complete manifold with asymptotically cylindrical ends.

The goal of this section is to make use of the surgery calculus of [16] and study the uniform behaviour of the Quillen metric and the Bismut-Freed connection when we pass from the determinant bundle associated to the family of Dirac operators fixed by the closed Riemannian fibration $\left(\psi: M \rightarrow B, g_{M / B}\left(\varepsilon_{0}\right)\right)$ to the $b$-determinant bundle associated to the family of Dirac operators fixed by the exact $b$-Riemannian fibration with boundary ( $\left.\bar{\psi}: \bar{M} \rightarrow B, g_{M / B}(0)\right)$.

Notice that the Hermitian geometry of $\operatorname{det}(ð), \partial$ being the original family, and $\operatorname{det}\left(ð\left(\varepsilon_{0}\right)\right)$ are easily related. These line bundles are obviously isomorphic; moreover they admit Quillen metrics and Bismut-Freed connections with curvatures equal to the 2-form piece of the right-hand side of the respective local family index formulae. By well known transgression formulae the two curvatures differ by an exact 2-form $d \alpha \in \mathscr{C}^{\infty}\left(B ; \Lambda^{2}\right)$. In particular by subtracting the globally defined 1 -form $\alpha$ it is always possible to modify the Bismut-Freed connection $\nabla^{\operatorname{det}}$ on $\operatorname{det}(ð)$ so as to define a new connection $\widetilde{\nabla}^{\text {det }}$ with curvature precisely equal to the one of the Bismut-Freed connections of $\operatorname{det}\left(ð\left(\varepsilon_{0}\right)\right)$. Thus there is no loss of generality in assuming that $ð=ð\left(\varepsilon_{0}\right)$.

Let us explain our goal more precisely. If the fibres of $\psi: M \rightarrow B$ have a spin structure, then, as explained in [16], the metrics (4.1) and the choice of a spin structure on $M_{z}, z \in B$ fix a surgery spin structure: this means that for each $z \in B$ there exists a well defined spinor bundle $S$ on $\left(M_{z}\right)_{s}=\left[M_{z} \times[0,1] ; H_{z} \times\{0\}\right]$, a Clifford action $\mathrm{cl}_{S}(\cdot)$ of ${ }^{s} T\left(M_{z}\right)_{s}$ on $S$ and an induced Levi-Civita connection $\nabla^{S}$. These data endow $S \rightarrow\left(M_{z}\right)_{s}$ with the structure of a surgery Hermitian Clifford module with Hermitian Clifford connection. The associated Dirac operator

$$
ð_{z}(\varepsilon)=\frac{1}{i} \operatorname{cl}_{S} \nabla^{S}
$$

is an element in $\operatorname{Diff}_{s}^{1}\left(M_{z} ; S\right)$ (see [16] for the notation). The latter statement simply specifies how the $\varepsilon$-family of the differential operator $\partial_{z}(\varepsilon)$ fixed by (4.1) degenerates as $\varepsilon \downarrow 0$; in particular the limit operator $ð_{z}(0)$ is, for each $z \in B$, an element in $\operatorname{Diff}_{b}^{1}\left(\bar{M}_{z} ; S\right)$. We will also use the notation $\delta_{z, \bar{M}}$ for the limit operator: notice that $\mathrm{\delta}_{z, \bar{M}}=\mathrm{\partial}_{z, \bar{M}^{0}} \oplus \mathrm{\partial}_{z, \bar{M}^{1}}$. 
It is important to understand precisely the structure of the operators $ð_{\bar{M}^{0}}, \partial_{\bar{M}^{1}}$ near the respective boundaries. We consider the bundle endomorphisms $L_{\varepsilon}^{+}=\operatorname{Id}$ and $L_{\varepsilon}^{-}=\operatorname{cl}\left(d x /\left(x^{2}+\varepsilon^{2}\right)^{\frac{1}{2}}\right)$. Let $\phi_{\varepsilon}^{1}, \ldots, \phi_{\varepsilon}^{n}, n=2 k$ be a $g(\varepsilon)$-orthogonal local basis in the vertical cotangent bundle with $\phi_{\varepsilon}^{1}=d x /\left(x^{2}+\varepsilon^{2}\right)^{\frac{1}{2}}$. Finally let $\Gamma_{\varepsilon}$ be the associated chirality operator. Thus $\Gamma_{\varepsilon}^{2}=\mathrm{Id}$ and the positive and negative spinors are by definition the eigenspaces associated to 1 and -1 respectively. Let $L_{0}^{ \pm}$and $\Gamma_{0}$ be the limit endomorphisms. Following [18] and using the grading induced by $\Gamma_{0}$ it is easy to see that near the boundary:

$$
L_{0}^{\mp} \cdot{\partial_{\bar{M}}^{ \pm}}^{ \pm} \cdot\left(L_{0}^{ \pm}\right)^{-1}= \pm x \frac{\partial}{\partial x}+{\partial_{H}}
$$

with $ð_{H}$ equal to the Dirac family on $\chi: H \rightarrow B$ induced by the boundary Clifford action

$$
\operatorname{cl}_{\partial}(\xi)=\operatorname{cl}\left(i \frac{d x}{x}\right) \operatorname{cl}(\xi) \quad \text { with } \xi \in T^{*}(H / B) \equiv T^{*}\left(\partial \bar{M}^{1} / B\right) .
$$

Similarly

$$
L_{0}^{\mp} \cdot \partial_{\bar{M}^{0}}^{ \pm} \cdot\left(L_{0}^{ \pm}\right)^{-1}= \pm y \frac{\partial}{\partial y}+\partial_{(-H)} \quad \text { with } y=-x .
$$

Following [16] we first make the following assumption:

$\forall z \in B$ the Dirac operator induced by $\varlimsup_{z, \bar{M}}$ on $H_{z}$ is invertible .

In Sect. 8 we shall relax assumption (4.4) and only assume that these operators have null space of constant rank in $z$.

To each family $ð(\varepsilon)=\left(\partial_{z}(\varepsilon)\right)_{z \in B}$, with $0<\varepsilon \leqq \varepsilon_{0}$, we can associate a determinant bundle with a Quillen metric and a Bismut-Freed connection. Moreover, by the results of the previous sections, the same can be done for the family of $b$-Dirac operators $\partial(0) \in \operatorname{Diff}_{b, \bar{\psi}}(\bar{M}, S)$ and the real interest now is to understand how these geometric objects degenerate when $\varepsilon \downarrow 0$. This explains the program of the present section whenever we are dealing with Dirac operators associated to spin structures as well as with their twisted versions.

In the general case, i.e. when the original family $\partial\left(\varepsilon_{0}\right)$ is made of a generalized Dirac operator acting on the sections of a vertical Clifford module $E\left(\varepsilon_{0}\right)$, we need to introduce on $E$, the pull-back of $E\left(\varepsilon_{0}\right)$ to the surgery space, a surgery Hermitian Clifford structure together with an Hermitian $s$-connection. Once a defining function for $H$ has been fixed there is an isomorphism between ${ }^{s} T\left(M_{z}\right)_{s}, z \in B$, and the pullback of $T M_{z}$ to $\left(M_{z}\right)_{s}$. Thus there certainly exists an action of $\mathrm{Cl}\left({ }^{s} T\left(M_{z}\right)_{s}\right)$ on $E$ (i.e. a surgery Clifford action $\left.\mathrm{cl}_{s}(\cdot)\right)$ and the problem is to fix on $E$ an Hermitian metric and an $s$-connection $\nabla$ so that $\mathrm{cl}_{s}(\cdot)$ is unitary and $\nabla$ is Hermitian and Clifford with respect to the Levi-Civita $s$-connection fixed by (4.1). By a partition of unity argument such a structure can always be fixed. To any such structure we can associate a generalized surgery Dirac operator $\mathrm{\partial}_{z}(\varepsilon) \in \operatorname{Diff}_{s}^{1}\left(M_{z} ; E\right)$. We can let $z$ vary obtaining $\varepsilon$-families of Dirac operators $\partial(\varepsilon)$ and the goal is once again to investigate the geometry of $\operatorname{det}(\partial(\varepsilon))$ uniformly as $\varepsilon \downarrow 0$.

Our first result concerns the limiting behaviour of $\operatorname{det}(\partial(\varepsilon))$, the determinant bundle itself. For each $\varepsilon \in\left(0, \varepsilon_{0}\right]$ the two families. $ठ\left(\varepsilon_{0}\right)$ are homotopic; thus the two associated determinant line bundles are isomorphic. We can specify this isomorphism and study its behaviour as $\varepsilon \downarrow 0$. 
Proposition 4. For each fixed $\varepsilon \in\left(0, \varepsilon_{0}\right]$ there exists a continuous family of natural isomorphisms

$$
S(\varepsilon, \bar{\varepsilon}): \operatorname{det}(ð(\varepsilon)) \rightarrow \operatorname{det}(ð(\bar{\varepsilon}))
$$

with $0<\bar{\varepsilon} \leqq \varepsilon$ which extends in a continuous fashion down to $\bar{\varepsilon}=0$, producing a natural isomorphism

$$
S(\varepsilon) \equiv S(\varepsilon, 0): \operatorname{det}(ð(\varepsilon)) \rightarrow \operatorname{det}_{b}(ð(0))=\operatorname{det}_{b}\left(\partial_{\bar{M}^{0}}\right) \otimes \operatorname{det}_{b}\left(\partial_{\bar{M}^{1}}\right) .
$$

Proof. We make heavy use of the results in [16] on the uniform structure of the resolvent of $\partial(\varepsilon)^{2}$ as $\varepsilon \downarrow 0$. Let $\delta$ be smaller than the first eigenvalue of $\partial_{z, H}$ for each $z \in B$ and let $\mathscr{U}=\left\{U_{\lambda}\right\}$ the open cover of $B$ defined as in Sect. 2 by the limit family $\partial^{2}(0) \equiv \chi_{\bar{M}}^{2}$. We first concentrate on an open set $W_{\lambda} \in \mathscr{U}$ on which

$$
\operatorname{spec}\left(ð_{z}^{2}(0)\right) \cap[0, \lambda)=0 \quad \forall z \in W_{\lambda} .
$$

Let $N^{ \pm}$be the dimensions of null $\left(\chi_{z}^{\mp}(0) \chi_{z}^{ \pm}(0)\right)$ and let $\Pi_{z}^{ \pm}(0)$ be the $L_{b}^{2}$-orthogonal projection onto these subspaces. According to Sect. 5 in [16] if $\varepsilon_{0}>0$ and $\lambda>0$ are chosen small enough the $g_{z}(\varepsilon)$-orthogonal projector $\Pi_{z}^{\lambda}(\varepsilon)$ onto the eigenfunctions associated to the eigenvalues of $\delta_{z}^{\mp}(\varepsilon) \delta_{z}^{ \pm}(\varepsilon)$ contained in the interval $[0, \lambda)$ is uniformly of finite rank exactly $N^{ \pm}$for each $\varepsilon \in\left[0, \varepsilon_{0}\right]$ and for each $z \in W_{\lambda}$.

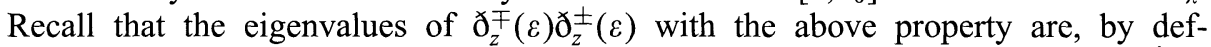
inition, the small eigenvalues; moreover for each $z \in W_{\lambda}$ these projections $\Pi_{z}^{\lambda}(\varepsilon)$ belong to $\Psi_{s, \tau}^{-\infty}, \tau>0$, the boundary terms in the surgery calculus. For the above choice of $\lambda$ and $\varepsilon_{0}$ we can assume that $\lambda \notin \operatorname{spec}\left(夭_{z}^{2}(\varepsilon)\right), \forall \varepsilon \leqq \varepsilon_{0}$ and $\forall z \in W_{\lambda}$. Thus

$$
\operatorname{det}(ð(\varepsilon)) \uparrow W_{\lambda}=\operatorname{det}\left(H_{[0, \lambda)}\right)=\operatorname{det}\left(\operatorname{ran} \Pi^{\lambda}(\varepsilon)\right) .
$$

Let $\left\{e_{k}^{ \pm}(\varepsilon)\right\}, k=1,2, \ldots, N^{ \pm}$be an orthonormal basis of eigenfunctions for ${ }_{z}^{\mp}(\varepsilon)$ $\partial_{z}^{ \pm}(\varepsilon)$ associated to the small eigenvalues. Let $\left\{\eta^{k, \pm}(\varepsilon)\right\}$ be the dual basis. For each $\bar{\varepsilon} \in(0, \varepsilon)$ we can define a bundle map

$$
S(\varepsilon, \bar{\varepsilon}): \operatorname{det}(ð(\varepsilon)) \uparrow W_{\lambda} \rightarrow \operatorname{det}(ð(\bar{\varepsilon})) \uparrow W_{\lambda}
$$

by associating to an element $v(\varepsilon) \in \operatorname{det}\left(\operatorname{ran} \Pi^{\lambda}(\varepsilon)\right)$

$$
v(\varepsilon)=a \eta^{+, 1}(\varepsilon) \wedge \cdots \wedge \eta^{+, N_{+}}(\varepsilon) \otimes e_{1}(\varepsilon) \wedge \cdots \wedge e_{N_{-}}(\varepsilon)
$$

the element

$$
S(\varepsilon, \bar{\varepsilon}) v(\varepsilon) \equiv a \eta^{+, 1}(\bar{\varepsilon}) \wedge \cdots \wedge \eta^{+, N_{+}}(\bar{\varepsilon}) \otimes e_{1}(\bar{\varepsilon}) \wedge \cdots \wedge e_{N_{-}}(\bar{\varepsilon})
$$

in $\operatorname{det}\left(\operatorname{ran} \Pi^{\lambda}(\varepsilon)\right)$. Since $\Pi_{z}^{\lambda}(\varepsilon) \in \Psi_{s, \tau}^{-\infty}, \tau>0$ for each $z \in W_{\lambda}$, it follows that $S(\varepsilon, \bar{\varepsilon})$ is continuous in $\bar{\varepsilon}$ in the $C^{0}$-norm of $\mathscr{C}^{\infty}\left(W_{\lambda} ; \operatorname{Hom}(\operatorname{det}(ð(\varepsilon)), \operatorname{det}(ð(\bar{\varepsilon})))\right)$. Moreover $S(\varepsilon, \bar{\varepsilon})$ extends continuously down to $\bar{\varepsilon}=0$ giving a bundle map:

$$
\begin{aligned}
& S(\varepsilon): \operatorname{det}(ð(\varepsilon)) \uparrow W_{\lambda} \rightarrow \Lambda^{\max }\left(\operatorname{ran} \Pi^{+, \lambda}(0)\right)^{*} \otimes \Lambda^{\max }\left(\operatorname{ran} \Pi^{-, \lambda}(0)\right) \\
& \quad=\operatorname{det}\left(\operatorname{null}{\varlimsup_{\bar{M}}}\right)=\left(\operatorname{det}_{b}\left({\varlimsup_{\bar{M}}}^{0}\right) \otimes \operatorname{det}_{b}\left({\varlimsup_{\bar{M}}}^{1}\right)\right) \uparrow W_{\lambda} .
\end{aligned}
$$

Since $\Pi^{ \pm, \lambda}(\varepsilon)$ is for $\varepsilon \in\left[0, \varepsilon_{0}\right]$ uniformly of rank $N^{ \pm}$, it follows that these maps are isomorphisms. Thus the proposition is proved for the restriction of the determinant line bundles to the open set $W_{\lambda}$. To handle the general case, i.e. without 
assuming (4.7), it suffices to show once again that if $\varepsilon_{0}$ is small enough and if $\lambda<\delta$ is properly chosen, then $\forall z \in U_{\lambda}, U_{\lambda} \in \mathscr{U}$, the $g_{z}(\varepsilon)$-projector $\Pi_{z}^{\lambda}(\varepsilon)$ onto the eigenfunctions of $\partial_{z}(\varepsilon)^{2}$ corresponding to eigenvalues less than $\lambda$ is uniformly of finite rank equal to $N$ (in turn equal to the sum of the multiplicities of the eigenvalues of $\partial_{z}(0)^{2}$ which are less than $\left.\lambda\right)$. The arguments of Sect. 5.3 in [16] certainly prove that $\operatorname{rk}\left(\Pi_{z}^{\lambda}(\varepsilon)\right) \geqq N$ : simply apply the argument there to show that $\forall \lambda_{j} \in \operatorname{spec}\left(\partial_{z}(0)^{2}\right), \quad \lambda_{j}<\lambda$, there exists a neighbourhood $V_{j} \subset \mathbb{R}$ such that the spectral projector $\Pi_{z}^{\lambda, j}(\varepsilon)$ corresponding to the eigenvalues of $ð_{z}(\varepsilon)^{2}$ contained in $V_{j}$ is, for $\varepsilon$ small enough, uniformly of rank equal to $N_{j}=$ multiplicity of $\lambda_{j}$. To show that $\operatorname{rk}\left(\Pi_{z}^{\lambda}(\varepsilon)\right)=\operatorname{rk}\left(\Pi_{z}^{\lambda}(0)\right)=N$ we need to rule out the existence of a family of eigenvalues $\mu(\varepsilon)$ for $ð^{2}(\varepsilon)$ such that $\lim \mu(\varepsilon)=\bar{\mu}<\lambda$ but $\bar{\mu} \notin \operatorname{spec}\left(ð^{2}(0)\right)$. To do so we can simply apply Proposition 2 of [16]. Alternatively a direct argument employing Weyl's criterion ([24]) can be given. The proposition is proved.

Let us denote by $\Pi_{\geqq}$the spectral projection corresponding to the non-negative eigenvalues of the boundary family of $\partial_{\bar{M}}$, i.e. the family $\partial_{H}$. Using (4.2), (4.3) we

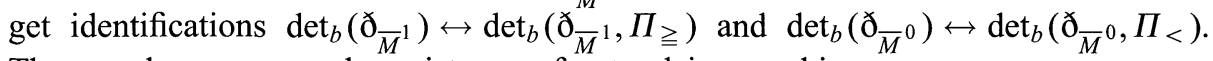
Thus we have proven the existence of natural isomorphisms

$$
S(\varepsilon): \operatorname{det}(ð(\varepsilon)) \rightarrow \operatorname{det}_{b}\left(\partial_{\bar{M}^{0}}, \Pi_{<}\right) \otimes \operatorname{det}_{b}\left(\partial_{\bar{M}^{1}}, \Pi_{\geqq}\right) .
$$

On the determinant line bundle $\operatorname{det}_{b}(ð(0)) \equiv \operatorname{det}_{b}\left(\partial_{\bar{M}^{0}}\right) \otimes \operatorname{det}_{b}\left(\partial_{\bar{M}^{1}}\right)$ we can consider two metrics. The $b$-Quillen metric $\|\cdot\|_{b, Q}$ defined by (2.5) and the pushforward, through the isomorphism

$$
S(\varepsilon): \operatorname{det}(ð(\varepsilon)) \rightarrow \operatorname{det}_{b}\left(\partial_{\bar{M}^{0}}\right) \otimes \operatorname{det}_{b}\left(\varlimsup_{\bar{M}^{1}}\right),
$$

of the Quillen metric $\|\cdot\|_{Q(\varepsilon)}$ on $\operatorname{det}(\partial(\varepsilon))$. We denote by $\|\cdot\|_{*, \varepsilon}$ this push-forward metric. It is somehow surprising that the metrics $\|\cdot\|_{*, \varepsilon}$ do not converge as $\varepsilon \downarrow 0$ to $\|\cdot\|_{b, Q}$. Nevertheless the following holds.

Proposition 5. Let $v \in \mathscr{C}^{\infty}\left(B ; \operatorname{det}_{b}\left({\partial_{\bar{M}^{0}}}\right) \otimes \operatorname{det}_{b}\left(\partial_{\bar{M}^{1}}\right)\right)$ and let $z \in B$. Then as $\varepsilon \downarrow 0$,

$$
\varepsilon^{\zeta^{\prime}\left(0, \partial_{z, H}^{2}\right)}\|v(z)\|_{*, \varepsilon} \rightarrow\|v(z)\|_{b, Q} \text {. }
$$

Proof. By definition

$$
\|v(z)\|_{*, \varepsilon}=\left\|(S(\varepsilon))^{-1} v(z)\right\|_{Q(\varepsilon)} .
$$

If we assume, as we can, that for $\varepsilon_{0}$ small enough and $\lambda<\delta, \lambda \notin \operatorname{spec}\left(ð^{2}(\varepsilon)\right)$ for $\varepsilon \in\left[0, \varepsilon_{0}\right]$, then

$$
\|v(z)\|_{*, \varepsilon}=e^{-\zeta^{\prime}\left(0, \partial_{z}^{-}(\varepsilon) \partial_{z}^{+}(\varepsilon), \lambda\right) / 2}\left|(S(\varepsilon))^{-1} v(z)\right|_{L^{2}\left(g_{z}(\varepsilon)\right)} .
$$

It follows from our definitions that

$$
\lim _{\varepsilon \downarrow 0}\left|(S(\varepsilon))^{-1} v(z)\right|_{L^{2}\left(g_{z}(\varepsilon)\right)}=|v(z)|_{L_{b}^{2}} .
$$

To understand the asymptotic behaviour of the term in (4.10) involving the zeta function, we use once again the results in [16] and more precisely the heat surgery calculus. Using the arguments given in Sects. 6 and 7 of [16] we see that as functions of $z \in U_{\lambda}$,

$$
\begin{aligned}
\zeta^{\prime}\left(0, \partial_{z}^{-}(\varepsilon) \partial_{z}^{+}(\varepsilon), \lambda\right)= & { }^{b \zeta^{\prime}}\left(0, \partial_{z}^{-}(0) \partial_{z}^{+}(0), \lambda\right)+\log \varepsilon \cdot 2 \zeta^{\prime}\left(0, \partial_{z, H}^{2}, \lambda\right) \\
& +r_{\lambda}(z, \varepsilon) \cdot \log \varepsilon+s_{\lambda}(z, \varepsilon)
\end{aligned}
$$


with $r_{\lambda}, s_{\lambda} \in \mathscr{C}^{\infty}\left(U_{\lambda} \times\left[0, \varepsilon_{0}\right]\right)$ and $r_{\lambda}(z, 0)=s_{\lambda}(z, 0)=0$ for each $z \in U_{\lambda}$. Notice that from Lemma 2 of [16] we would get, a priori, an extra term in (4.11) involving the $b$-trace of the surgery normal operator of the heat kernel; this term is always zero because of the $\mathbb{R}^{+}$-invariance. From (4.11) the proposition follows since $\lambda<\delta$ and $\operatorname{spec}\left(\Varangle_{z, H}^{2}\right) \cap[0, \delta)=\emptyset$, so that $\zeta^{\prime}\left(0, ð_{z, H}^{2}, \lambda\right)=\zeta^{\prime}\left(0, ð_{z, H}^{2}\right)$.

It should be remarked that although from the odd-dimensionality of $H_{z}$ it follows that $\zeta\left(0, \partial_{z, H}^{2}\right)=0$ there is no reason to expect $\zeta^{\prime}\left(0, \partial_{z, H}^{2}\right)$ to be zero.

We now pass to the study of the asymptotic behaviour of the (push-forward of the) Bismut-Freed connection $\nabla^{\operatorname{det}, \varepsilon}$ on $\operatorname{det}(\partial(\varepsilon))$. By the results of the previous sections we can introduce on $\operatorname{det}_{b}(ð(0))=\operatorname{det}_{b}\left(\varlimsup_{\bar{M}}\right)$, a Bismut-Freed connection for which we keep the notation ${ }^{b} \nabla^{\text {det }}$. We will denote by ${ }^{b} \nabla^{\text {det, } 0}$ and ${ }^{b} \nabla^{\text {det, } 1}$ the analogous Bismut-Freed connections on $\operatorname{det}_{b}\left(\partial_{\bar{M}^{0}}\right), \operatorname{det}_{b}\left({\partial_{\bar{M}}}^{1}\right)$.

For $\varepsilon>0$ let us consider the metric

$$
\|\cdot\|_{\#, \varepsilon}=\varepsilon^{\zeta^{\prime}\left(0, \partial_{H}^{2}\right)}\|\cdot\|_{*, \varepsilon}
$$

on $\operatorname{det}_{b}(\partial(0))$. Let $\nabla^{*, \varepsilon}$ the push-forward of the Bismut-Freed connection on $\operatorname{det}(ð(\varepsilon))$ through the isomorphism $S(\varepsilon)$. This is a connection on $\operatorname{det}_{b}(ð(0))$ compatible with $\|\cdot\|_{*, \varepsilon}$. According to Proposition 9.10 in [2] the connection $\nabla^{*, \varepsilon}+$ $d \zeta^{\prime}\left(0, ð_{H}^{2}\right) \cdot \log \varepsilon$ is compatible with $\|\cdot\|_{\#, \varepsilon}$. Since by Proposition $5\|\cdot\|_{\#, \varepsilon} \rightarrow$ $\|\cdot\|_{b, Q}$ as $\varepsilon \downarrow 0$ we can at once guess the following.

\section{Proposition 6. Let}

$$
\nabla^{*, \varepsilon}: \mathscr{C}^{\infty}\left(B, \operatorname{det}_{b}\left(\varlimsup_{\bar{M}^{0}}\right) \otimes \operatorname{det}_{b}\left({\partial_{\bar{M}^{1}}}\right)\right) \rightarrow \mathscr{C}^{\infty}\left(B, \operatorname{det}_{b}\left(\varlimsup_{\bar{M}^{0}}\right) \otimes \operatorname{det}_{b}\left({\partial_{\bar{M}^{1}}}\right) \otimes T^{*} B\right)
$$

be the push-forward of the Bismut-Freed connection $\nabla^{\operatorname{det}, \varepsilon}$ on $\operatorname{det}(ð(\varepsilon))$ through the isomorphism (4.6). Then as $\varepsilon \downarrow 0$ we have

$$
\nabla^{*, \varepsilon}+d \zeta^{\prime}\left(0, \partial_{H}^{2}\right) \cdot \log \varepsilon \rightarrow{ }^{b} \nabla^{\operatorname{det}} .
$$

The convergence of two connections is to be understood as the $C^{k}$-convergence of the connection one-forms associated to any trivializing neighbourhood.

Proof. To rigorously prove (4.12) recall the definition of the Bismut-Freed connection $\nabla^{\operatorname{det}, \varepsilon}$ on $\operatorname{det}(ð(\varepsilon)) \uparrow U_{\lambda}=\operatorname{det}\left(H_{[0, \lambda)}(\varepsilon)\right)$, with $\lambda$ chosen as in Proposition 5. By definition $\nabla^{\text {det, } \varepsilon}=\nabla^{\lambda, \varepsilon}+\beta_{\varepsilon}^{+}(\lambda)$ with $\beta_{\varepsilon}^{+}(\lambda)$ defined as in (3.1), (3.3) but with the trace functional replacing the $b$-trace functional. Thus

$$
\beta_{\varepsilon}^{+}(\lambda)=2 \underset{s \rightarrow 0}{\operatorname{LIM}} \int_{s}^{\infty} \operatorname{Tr}_{+}\left(F_{\varepsilon}^{+}(\lambda, t)\right) d t
$$

where, by definition,

$$
F_{\varepsilon}^{ \pm}(\lambda, t)=\left(-\frac{1}{2} \operatorname{Tr}_{ \pm}\left(P_{(\lambda, \infty)} \partial^{\mp}(\varepsilon)\left[\mathbb{A}_{[1]}(\varepsilon), \partial^{ \pm}(\varepsilon)\right] e^{-t(\partial(\varepsilon))^{2}}\right)\right)_{[1]} .
$$

Hence on $U_{\lambda}$ we have $\nabla^{*, \varepsilon}=\nabla^{*, \varepsilon, \lambda}+\beta_{\varepsilon}^{+}(\lambda)$ with $\nabla^{*, \varepsilon, \lambda}$ equal to the connection obtained by pushing forward the connection $\nabla^{\lambda, \varepsilon}$ on $\operatorname{det}\left(H_{[0, \hat{\lambda})}(\varepsilon)\right)$ through the isomorphism $S(\varepsilon)$. Remark now that $\nabla^{\lambda, \varepsilon}$ is induced by the connection obtained by compressing onto $H_{[0, \lambda)}(\varepsilon)$, the one-form piece of the Bismut superconnection $\mathbb{A}(\varepsilon)$ through the orthogonal projection $P_{[0, \lambda)}(\varepsilon)$. From the proof of Proposition 5 , i.e. ultimately from the uniform behaviour of the spectral projections $P_{[0, \lambda)}(\varepsilon)$, it follows in a straightforward way that $\nabla^{*, \varepsilon, \lambda} \rightarrow{ }^{b} \nabla^{\lambda}$ as $\varepsilon \downarrow 0$. Hence it suffices 
to analyze the asymptotic behaviour, in $\varepsilon$, of the one-form $\beta_{\varepsilon}^{+}(\lambda) \in \mathscr{C}^{\infty}\left(U_{\lambda}, \Lambda^{1}\right)$. Applying the notation and the results of [16] we have as in the proof of Proposition 5,

$$
\begin{aligned}
\beta_{\varepsilon}^{+}(\lambda)= & \beta_{b}^{+}(\lambda)+2 \underset{s \rightarrow 0}{\operatorname{LIM}} \int_{s}^{\infty} b-\operatorname{Tr}\left(N_{s}\left(F_{\varepsilon}^{+}(\lambda, t)\right)\right) d t \\
& +\log \varepsilon \cdot 2 \underset{s \rightarrow 0}{\operatorname{LIM}} \int_{s}^{\infty} \operatorname{Tr}\left(R_{H}\left(F_{\varepsilon}^{+}(\lambda, t)\right)\right) d t+v_{\lambda}(\varepsilon) \cdot \log \varepsilon+w_{\lambda}(\varepsilon)
\end{aligned}
$$

with $v_{\lambda}, w_{\lambda} \in \mathscr{C}^{\infty}\left(U_{\lambda} \times\left[0, \varepsilon_{0}\right] ; \Lambda^{1}\right), v_{\lambda}(z, 0)=w_{\lambda}(z, 0)=0 \forall z \in U_{\lambda}$ and with $N_{s}$ and $R_{H}$ denoting the normal homomorphisms of [16]. Once again the term involving the surgery normal operator of $F_{\varepsilon}^{+}(\lambda, t)$, visualizing,

$$
2 \underset{s \rightarrow 0}{\operatorname{LIM}} \int_{s}^{\infty} b-\operatorname{Tr}\left(N_{s}\left(F_{\varepsilon}^{+}(\lambda, t)\right)\right) d t
$$

is equal to zero because of the $\mathbb{R}^{+}$-invariance of $N_{S}(ð(\varepsilon))$. Let $\widetilde{H}$ be the boundary of $\bar{M}$ (i.e. the disjoint union of two copies of $H$ ). Since by definition $R_{H}\left({ }^{ \pm}(\varepsilon)\right)=\mathrm{\partial}_{\widetilde{H}}$, we obtain

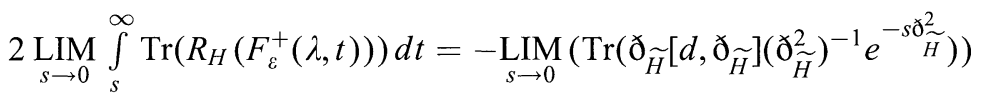

$$
\begin{aligned}
& =-\frac{1}{2} \underset{s \rightarrow 0}{\operatorname{LIM}}\left(\operatorname{Tr}\left(d ð_{\widetilde{H}}^{2}\left(ð_{\widetilde{H}}^{2}\right)^{-1} e^{-s ð_{\widetilde{H}}^{2}}\right)\right)=-\frac{1}{2} d \zeta^{\prime}\left(0, ð_{\widetilde{H}}^{2}\right) .
\end{aligned}
$$

From this last formula and (4.13) it follows that

$$
\lim _{\varepsilon \downarrow 0}\left(\beta_{\varepsilon}^{+}(\lambda)+\frac{1}{2} \log \varepsilon \cdot d \zeta^{\prime}\left(\partial_{\widetilde{H}}^{2}, 0\right)\right)=\beta_{b}^{+}(\lambda),
$$

and since $d \zeta^{\prime}\left(ð_{\widetilde{H}}^{2}, 0\right)=2 d \zeta^{\prime}\left(\mho_{H}^{2}, 0\right)$, the proposition follows for the $C^{0}$-convergence. Using Duhamel's principle the same arguments can be used to prove $C^{k}$ convergence.

\section{Section 5. Additivity Formulae for Local and Global Anomalies}

In this section we will draw two important corollaries from the results of Sect. 4.

Proposition 7. Let $R_{\operatorname{det}}(\varepsilon) \in \mathscr{C}^{\infty}\left(B ; \Lambda^{2}\right)$ be the curvature of the Bismut-Freed connection on $\operatorname{det}(ð(\varepsilon))$ and let $R_{\operatorname{det}_{b}}^{0}, R_{\operatorname{det}_{b}}^{1} \in \mathscr{C}^{\infty}\left(B ; \Lambda^{2}\right)$ respectively the curvatures of the Bismut-Freed connections on $\operatorname{det}_{b}\left(\partial_{\bar{M}^{0}}\right)$ and $\operatorname{det}_{b}\left(\partial_{\bar{M}^{1}}\right)$. Then as $\varepsilon \downarrow 0$

$$
R_{\operatorname{det}}(\varepsilon) \rightarrow R_{\operatorname{det}_{b}}^{0}+R_{\operatorname{det}_{b}}^{1} .
$$

Notice that since we are now comparing curvatures, i.e. elements in $\mathscr{C}^{\infty}\left(B ; \Lambda^{2}\right)$, we do not need to take the push-forward of the Bismut-Freed connection on $\operatorname{det}(ð(\varepsilon))$ through the isomorphism $S(\varepsilon)$. The limit (5.1) holds with respect to any $C^{k}$-norm.

Proof. Let ${ }^{b} \nabla^{\text {det }}$ be the Bismut-Freed connection on $\operatorname{det}_{b}\left(\delta_{\bar{M}}\right)$. For $\lambda<\delta, \delta$ smaller than the first non-zero eigenvalue of $\partial_{z, H} \forall z$, consider the open set $U_{\lambda}=$ $\left\{z \in B ; \lambda \notin \operatorname{spec}\left(\partial_{\bar{M}}\right)\right\}$. For small enough we have $U_{\lambda} \subset\left\{z \in B ; \lambda \notin \operatorname{spec}(ð(\varepsilon)) \forall_{\varepsilon}\right\}$. 
We prove convergence on the restriction of the determinant line bundles to $U_{\lambda}$. On $U_{\lambda}$ the two connections in question will be equal to $\nabla^{\lambda, \varepsilon}+\beta_{\varepsilon}^{+}(\lambda)$ and ${ }^{b} \nabla^{\lambda}+$ $\beta_{b}^{+}(\lambda)$ respectively. Taking the square of the two connections we obtain respectively $\left(\nabla^{\lambda, \varepsilon}\right)^{2}+d \beta_{\varepsilon}^{+}(\lambda)$ and $\left({ }^{b} \nabla^{\lambda}\right)^{2}+d \beta_{b}^{+}(\lambda)$. The results of the previous section, and more precisely (4.13), (4.14) show that $d \beta_{\varepsilon}^{+}(\lambda) \rightarrow d \beta_{b}^{+}(\lambda)$ as $\varepsilon \downarrow 0$. Since from our set-up it is clear, as in Proposition 6 , that $\left(\nabla^{\lambda, \varepsilon}\right)^{2} \rightarrow\left({ }^{b} \nabla^{\lambda}\right)^{2}$, the proposition is proved.

Formula (5.1) gives the asymptotic additivity of the curvatures of the BismutFreed connections on $\operatorname{det}(ð(\varepsilon))$ and $\operatorname{det}_{b}\left(\partial_{\bar{M}^{0}}\right) \otimes \operatorname{det}_{b}\left(\partial_{\bar{M}^{1}}\right)$. This also follows from the local anomaly formula proved in the next section. To give a more interesting application, consider the holonomy of these connections around a loop $\gamma \subset B$. Since the extra term in formula (4.14) is exact, we have, directly from the proof of Proposition 6,

Proposition 8. The holonomy of the Bismut-Freed connection on $\operatorname{det}(\Varangle(\varepsilon))$ around the loop $\gamma \subset B$ converges as $\varepsilon \downarrow 0$ to the product of the holonomies of the BismutFreed connections on $\operatorname{det}_{b}\left(ð_{\bar{M}^{0}}\right), \operatorname{det}_{b}\left(\partial_{\bar{M}^{1}}\right)$ :

$$
\operatorname{hol}_{\gamma}\left(\nabla^{\operatorname{det}, \varepsilon}\right) \rightarrow \operatorname{hol}_{\gamma}\left({ }^{b} \nabla^{\operatorname{det}, 0}\right) \cdot \operatorname{hol}_{\gamma}\left({ }^{b} \nabla^{\operatorname{det}, 1}\right) .
$$

\section{Section 6. The Local Anomaly Formula on Manifolds With Boundary}

We go back to the setting considered in Sects. 1,2 and 3. Thus we are given a fibration with boundary $\phi: M \rightarrow B$, a vertical Hermitian Clifford module $E$ with an Hermitian Clifford connection and the associated family of generalized Dirac operator $ð=\left(\partial_{z}\right) \in \operatorname{Diff}_{b, \phi}^{1}(M ; E)$. Under assumption (2.1) which will be made throughout this section we have defined on the determinant line bundle $\operatorname{det}_{b}(ð)$ associated to the induced family of Fredholm operators $\mathrm{\delta}_{z}: H_{b}^{1}\left(M_{z} ; E\right) \rightarrow L^{2}\left(M_{z} ; E\right)$ a $b$-Quillen metric $\|\cdot\|_{b, Q}$ and the analogue of the Bismut-Freed connection, ${ }^{b} \nabla^{\text {det }}$.

In order to sharpen (1.4) to a local formula for the curvature of ${ }^{b} \nabla^{\text {det }}$, recall the transgression formula (4) for our family of generalized Dirac operators. We apply this formula to the family $ð_{(\lambda, \infty)}$ on the set $U_{\lambda}$. The null bundle of $ð_{(\lambda, \infty)}$ is precisely $H_{[0, \lambda)}$ so that (4) reads

$$
\operatorname{Ch}\left(H_{[0, \lambda)}\right)=\frac{1}{(2 \pi i)^{\frac{n}{2}}} \int_{M / B} \widehat{A}(M / B) \operatorname{Ch}^{\prime}(E)-\frac{1}{2} \widehat{\eta}+d \int_{0}^{\infty} b-\operatorname{STr}\left(\frac{d \mathbb{A}_{s, \lambda}}{d s} e^{-\mathbb{A}_{s, \lambda}^{2}}\right) d s
$$

with $\mathbb{A}_{s, \lambda}=s^{\frac{1}{2}}{\partial_{(\lambda, \infty)}}+\mathbb{A}_{[1]}+s^{-\frac{1}{2}} \mathbb{A}_{[2]}$. The Chern character on the left-hand side is computed with respect to the connection $P_{[0, \lambda)} \mathbb{A}_{[1]} P_{[0, \lambda)}$. The fact that the boundary contribution remains unchanged for $ठ$ and $\delta_{(\lambda, \infty)}$ is again a consequence of the fact that $I\left(P_{[0, \lambda)}, w\right)=0$. It follows from formula $(6.1)$, as in [2], that

$$
\frac{1}{2} d\left(\beta^{+}(\lambda)-\beta^{-}(\lambda)\right)+\operatorname{Ch}\left(H_{[0, \lambda)}\right)_{[2]}=\left(\frac{1}{(2 \pi i)^{\frac{n}{2}}} \int_{M / B} \widehat{A}(M / B) \mathrm{Ch}^{\prime}(E)-\frac{1}{2} \widehat{\eta}\right)_{[2]} .
$$

This formula, together with Lemma 6 and the remark $\operatorname{Ch}\left(H_{[0, \lambda)}\right)_{[2]}=\left(\nabla^{\hat{\lambda}}\right)^{2}$, implies as in [2] the following result. 
Proposition 9. Let ठ be a family of Dirac operators as in Sect. 1 and let us make assumption (2.1). Let ${ }^{b} \nabla^{\mathrm{det}}$ be the connection on the determinant line bundle $\operatorname{det}_{b}\left(ð, \Pi_{\geqq}\right)$introduced in (3.4). Then

$$
\left({ }^{b} \nabla^{\operatorname{det}}\right)^{2}=\left(\frac{1}{(2 \pi i)^{\frac{n}{2}}} \int_{M / B} \widehat{A}(M / B) \mathrm{Ch}^{\prime}(E)-\frac{1}{2} \widehat{\eta}\right)_{[2]} .
$$

The asymptotic additivity of the curvature under surgery, as stated in Proposition 7, can also be proved directly using the local anomaly formula in the closed case together with Proposition 9. In fact from the structure of the families $\delta_{\bar{M}^{0}}, \delta_{\bar{M}^{1}}$ near the boundary, as given in (4.2), (4.3), we see that the two eta-form contributions in (6.3) have opposite signs, and hence cancel out when we add the two curvatures. Thus

$$
R_{\operatorname{det}_{b}}^{0}+R_{\operatorname{det}_{b}}^{1}=\frac{1}{(2 \pi i)^{\frac{n}{2}}} \int_{M^{0} / B} \widehat{A}(M / B) \mathrm{Ch}^{\prime}(E)+\frac{1}{(2 \pi i)^{\frac{n}{2}}} \int_{M^{1} / B} \widehat{A}(M / B) \mathrm{Ch}^{\prime}(E),
$$

from which the claim follows.

\section{Section 7. The Constant Rank Case: Spectral Cuts and Variation Formulae}

Before tackling the constant rank case we need some results on the analysis of elliptic families on manifolds with boundary.

Let $\phi: M \rightarrow B$ be a fibration of exact $b$-manifolds as in Sect. 1 and let us make assumption (1.5). If $r \in \mathbb{R}^{+}$is chosen small enough then, clearly, $-r \notin \operatorname{spec}\left(\partial_{0, z}\right)$ for each $z \in B$. The constant function $\tau(z)=r$ is then a spectral cut for the family $ð_{0}$ in the following sense:

Definition 1. Let $D=\left(D_{z}\right)$ be a smooth family of self-adjoint differential operators on closed compact manifolds. A spectral cut for $D$ is a function $\tau \in \mathscr{C}^{\infty}(B ; \mathbb{R})$ such that

$$
-r(z) \notin \operatorname{spec}\left(D_{z}\right) \quad \forall z \in B .
$$

As an example assume that there exists a smooth function $\theta \in \mathscr{C}^{\infty}(B ; \mathbb{R})$ such that the dimension of null $\left(D_{z}+\theta(z)\right)$ is constant. This implies that null $(D+\theta)$ is a smooth vector bundle and that $\tau(z)=\theta(z)+\varepsilon$ is a spectral cut for $\varepsilon>0$ small enough (in fact for $\varepsilon$ smaller than the minimum over $z \in B$ of the distance between $\theta(z)$ and $\left.\operatorname{spec}\left(D_{z}\right) \backslash\{\theta(z)\}\right)$. The case $\theta \equiv 0$ is particularly important for us; it applies for example to the boundary operators associated to a family of signature operators.

Let $\tau$ be a spectral cut for $\partial_{0}$. Let $P_{z}=\Pi_{\geqq-r}^{z}$ be the $L^{2}$-spectral projection corresponding to the eigenvalues of $\partial_{0, z}$ which are greater than $-\tau(z)$. Then $P$ is a spectral section; the determinant bundle $\operatorname{det}_{b}(ð, P)$ is associated to the global family of Fredholm operators

$$
\mathrm{\partial}_{z}^{+}: x^{\tau(z)} H_{b}^{1}\left(M_{z} ; E_{z}^{+}\right) \rightarrow x^{\tau(z)} L_{b}^{2}\left(M_{z} ; E_{z}^{-}\right)
$$

with cokernel naturally isomorphic to the null space of

$$
ð_{z}^{-}: x^{-\tau(z)} L^{2}\left(M_{z} ; E_{z}^{-}\right) \rightarrow x^{-\tau(z)} H_{b}^{-1}\left(M_{z} ; E_{z}\right) .
$$


We shall refer to $\tau(z)$ as a weight for ${ }_{z}^{+}$. If we consider the conjugated family $ð(\tau) \in \operatorname{Diff}_{b, \phi}^{1}(M ; E)$ defined as

$$
ð(\tau)_{z}=\left(\begin{array}{cc}
0 & x^{\tau(z)}{\varlimsup_{z}^{-}}^{-\tau(z)} \\
x^{-\tau(z)}{\oint_{z}^{+}}^{\tau} x^{\tau(z)} & 0
\end{array}\right),
$$

then the null space of (7.1) and (7.2) are given by the null spaces of $\partial(\tau)_{z}^{+}$and $ð(\tau)_{z}^{-}$acting on unweighted Sobolev spaces so that

$$
\operatorname{det}_{b}\left(ð, \Pi_{\geqq-\tau}\right)=\operatorname{det}_{b}\left(ð(\tau), \Pi_{\geqq}\right) \leftrightarrow \operatorname{det}_{b}(ð(\tau)) .
$$

More generally $\operatorname{Ind}\left(ð, \Pi_{\geqq-\tau}\right)=\operatorname{Ind}\left(ð(\tau), \Pi_{\geqq}\right)$in $K^{0}(B)$. Notice that

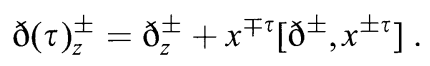

Recall that the local anomaly formula can be seen as an application of the transgressed form of the family index theorem. Let us thus see how the results of [18] specialize when we consider $\operatorname{Ind}\left(ð, \Pi_{\geqq-\tau}\right)=\operatorname{Ind}\left(ð(\tau), \Pi_{\geqq}\right)$.

Let $\mathbb{A}(\tau)=ð(\tau)+\mathbb{A}_{[1]}+\mathbb{A}_{[2]}$ be the Bismut superconnection associated to $\delta(\tau)$. The family $\partial(\tau)^{2} \in \operatorname{Diff}_{b, \phi}^{2}(M ; E)$ has a well defined heat kernel (since $\sigma_{\mathrm{pr}}\left(ð(\tau)^{2}\right)=$ $\left.\sigma_{\mathrm{pr}}\left(ð^{2}\right)\right)$; using Duhamel's expansion we can thus consider $\exp \left(-\mathbb{A}_{t}(\tau)^{2}\right)$ and its $b$-supertrace, with values in $\mathscr{C}^{\infty}\left(B, \Lambda^{*} B\right), b$-STr$\left(\exp \left(-\mathbb{A}_{t}(\tau)^{2}\right)\right) \equiv b$-Ch $\left(\mathbb{A}_{t}(\tau)\right)$. Recalling that for any smooth family of $b$-pseudodifferential operators $P \in \Psi_{b, \phi}^{*}(M ; E)$ one has

$$
I\left(x^{-w} P x^{w}, \lambda\right)=I(P, \lambda-i w) \quad \forall w \in \mathbb{C},
$$

we obtain, following the notation of [17],

$$
I(\mathbb{A}(\tau), \lambda)=\gamma \lambda+\sigma\left(ð_{0}+\tau\right)+\mathbb{B}_{[1]}+\sigma \mathbb{B}_{[2]} .
$$

Using the defect formula for the $b$-supertrace of a supercommutator as in [18] we get the fundamental formula

$$
\frac{d}{d t} b-\operatorname{Ch}\left(\mathbb{A}_{t}(\tau)\right)=-d_{B} b-\operatorname{STr}\left(\frac{d \mathbb{A}_{t}(\tau)}{d t} e^{-\mathbb{A}_{t}(\tau)^{2}}\right)-\frac{1}{2} \widehat{\eta}_{\tau}(t),
$$

where the eta forms are defined by

$$
\widehat{\eta}_{\tau}(t)=\frac{1}{\sqrt{\pi}} \operatorname{STr}_{\mathrm{Cl}(1)}\left(\frac{d \mathbb{B}_{t}(\tau)}{d t} e^{-\mathbb{B}_{t}(\tau)^{2}}\right)
$$

with $\mathbb{B}(\tau)=\sigma\left(ð_{0}+\tau\right)+\mathbb{B}_{[1]}+\sigma \mathbb{B}_{[2]}$. Let $\delta_{t}$ be the operator on $\mathscr{C}^{\infty}\left(B, \Lambda^{*} B\right)$ which acts as multiplication by $t^{-\frac{1}{2}}$ on $\mathscr{C}^{\infty}\left(B ; \Lambda^{i} B\right)$; let us define a differential form $\widehat{\operatorname{AS}}(\tau)$ on $M$ by

$$
\widehat{\mathrm{AS}}(\tau)(x)=\operatorname{LIM}_{t \downarrow 0}\left(\delta_{t}\left(\operatorname{str}_{E}\left(e^{-t \mathbb{A}(\tau)^{2}}\right)(x, x)\right)\right),
$$

and let

$$
\widehat{\eta}_{\tau}=\operatorname{LIM}_{t \downarrow 0} \int_{t}^{\infty} \widehat{\eta}_{\tau}(s) d s
$$

Since $-\tau(z) \notin \operatorname{spec}\left({\varlimsup_{0, z}}\right)$ the form $\widehat{\eta}_{\tau}(s)$ is exponentially converging to zero in any $C^{k}$-norm as $s \rightarrow \infty$. Thus the form $\widehat{\eta}_{\tau}$ is well defined. More generally if 
$\theta \in \mathscr{C}^{\infty}(B ; \mathbb{R})$ is such that null $\left(\partial_{0}+\theta\right)$ is a smooth vector bundle then, for $s$ large, we can give the following $C^{k}$-estimate:

$$
\left\|\operatorname{STr}_{\mathrm{Cl}(1)}\left(\frac{d \mathbb{B}_{s}(\theta)}{d s} e^{-\mathbb{B}_{s}(\theta)^{2}}\right)\right\|_{k} \leqq C(k) s^{-\frac{3}{2}},
$$

see $[8,2]$, and the eta form $\widehat{\eta}_{\theta}$ is again well defined.

The reason to take the regularized limits in (7.7), (7.8) is that the Dirac operators $ð(\tau)$ and $\partial_{0}+\tau$ are not associated to graded Hermitian Clifford connections.

Formula (7.5) can now be used to show that

$$
\operatorname{Ch}\left(\operatorname{Ind}\left(ð, \Pi_{\geqq-\tau}\right)\right)=\left[\int_{M / B}^{v} \widehat{\operatorname{AS}}(\tau)-\frac{1}{2} \widehat{\eta}_{\tau}\right] \quad \text { in } H^{*}(B) \text {. }
$$

Here the regularized integral of [17] appears; in the sequel we shall use the notation

$$
\operatorname{AS}(\tau)=\int_{M / B}^{v} \widehat{\operatorname{AS}}(\tau)
$$

Our real interest though is when the null spaces of $\partial(\tau)_{z}$ form a smooth vector bundle over $B$. If null $(\partial(\tau))$ is such a bundle and we denote by $\nabla^{\text {null }}$ the compression of $\mathbb{A}_{[1]}$ onto it, then, by applying formula (7.5), we obtain the following equality of differential forms:

$$
d \alpha_{b}(\tau)+\operatorname{Ch}\left(\operatorname{null} ð(\tau), \nabla^{\text {null }}\right)=\operatorname{AS}(\tau)-\frac{1}{2} \widehat{\eta}_{\tau}
$$

with

$$
\alpha_{b}(\tau)=\operatorname{LIM}_{t \downarrow 0} \int_{t}^{\infty} d_{B} b-\operatorname{STr}\left(\frac{d \mathbb{A}_{s}(\tau)}{d s} e^{-\mathbb{A}_{s}(\tau)^{2}}\right) d s .
$$

To bring this formula to a more useful form we study the variation of the terms on the right-hand side under a change of spectral cut. The formulae we get, together with those presented in [18], are of some independent interest.

First notice that $b$-Ch $\left(\mathbb{A}_{t}(\tau)\right)$ and $\widehat{\eta}_{\tau}(t)$ are in fact defined for each $\tau \in$ $\mathscr{C}^{\infty}(B ; \mathbb{R})$; it is only when we take the limit as $t \rightarrow \infty$ that the Fredholm properties of the family $\partial$, and hence the assumption that $\tau$ be a spectral cut, play a role.

Suppose that $\tau_{0}, \tau_{1} \in \mathscr{C}^{\infty}(B ; \mathbb{R})$, with $\tau_{1}(z)>\tau_{0}(z)$ for each $z \in B$. Let $\tau_{r} \in$ $\mathscr{C}^{\infty}(B ; \mathbb{R}), r \in[0,1]$, be a smooth homotopy connecting them.

Lemma 3. The $b$-Chern character of $\mathbb{A}_{t}\left(\tau_{r}\right)$ depends smoothly on $r$ and

$$
\frac{d}{d r} b-\operatorname{Ch}\left(\mathbb{A}_{t}\left(\tau_{r}\right)\right)=-d_{B} b-\operatorname{STr}\left(\frac{d \mathbb{A}_{t}\left(\tau_{r}\right)}{d r} e^{-\mathbb{A}_{t}\left(\tau_{r}\right)^{2}}\right)-\frac{t^{\frac{1}{2}}}{2 \sqrt{\pi}} \operatorname{STr}_{\operatorname{Cl}(1)}\left(\sigma \dot{\tau}_{r} e^{-\mathbb{B}_{t}\left(\tau_{r}\right)^{2}}\right)
$$

Proof. Recall that $[ð, f]=\operatorname{cl}(d f)$. Writing $\left(ð\left(\tau_{r}\right)\right)^{ \pm}$as $ð^{ \pm}+x^{\mp \tau_{r}}\left[ð^{ \pm}, x^{ \pm \tau_{r}}\right]$ we obtain, near the boundary of $M$,

$$
\frac{d \mathbb{A}_{t}\left(\tau_{r}\right)}{d r}=t^{\frac{1}{2}}\left(\begin{array}{cc}
0 & i \dot{\tau}_{r} \operatorname{cl}\left(\frac{d x}{x}\right) \\
-i \dot{\tau}_{r} \operatorname{cl}\left(\frac{d x}{x}\right) & 0
\end{array}\right)
$$

so that, using the identification $E_{\partial M} \leftrightarrow E^{0} \oplus E^{0}$ of [17], we obtain

$$
I\left(\frac{d \mathbb{A}_{t}\left(\tau_{r}\right)}{d r}, \lambda\right)=t^{\frac{1}{2}} \sigma \dot{\tau}_{r}
$$


Applying the defect formula for the $b$-supertrace of a supercommutator the proposition follows easily.

Taking the integral in $r$ from 0 to 1 and considering the regularized limit as in (7.7) we obtain from this lemma the following result:

$$
\begin{aligned}
\operatorname{AS}\left(\tau_{1}\right)-\operatorname{AS}\left(\tau_{0}\right)= & -d_{B} \int_{0}^{1} \operatorname{LiM}_{t \downarrow 0} b-\operatorname{STr}\left(\frac{d \mathbb{A}_{t}\left(\tau_{r}\right)}{d r} e^{-\mathbb{A}_{t}\left(\tau_{r}\right)^{2}}\right) d r \\
& -\int_{0}^{1} \operatorname{LIM}_{t \downarrow 0} \frac{t^{\frac{1}{2}}}{2 \sqrt{\pi}} \operatorname{STr}_{\mathrm{Cl}(1)}\left(\sigma \dot{\tau}_{r} e^{-\mathbb{B}_{t}\left(\tau_{r}\right)^{2}}\right) d r
\end{aligned}
$$

for any $\tau_{0}, \tau_{1} \in \mathscr{C}^{\infty}(B ; \mathbb{R})$.

Next we analyse the variation of $\widehat{\eta}_{\tau_{r}}(s)$ in $r$. Directly from Sect. 14 of [18] we have

Lemma 4. The differential forms $\widehat{\eta}_{\tau_{r}}(s)$ depend smoothly on $r$ and

$$
\frac{d}{d r} \widehat{\eta}_{\tau_{r}}(s)=\frac{d}{d s}\left(\frac{s^{\frac{1}{2}}}{\sqrt{\pi}} \operatorname{STr}_{\mathrm{Cl}(1)}\left(\sigma \dot{\tau}_{r} e^{-\mathbb{B}_{s}\left(\tau_{r}\right)^{2}}\right)\right)-d_{B} \operatorname{STr}_{\mathrm{Cl}(1)}\left(\sigma \dot{\tau}_{r} \frac{s^{\frac{1}{2}}}{\sqrt{\pi}} \frac{d \mathbb{B}_{s}\left(\tau_{r}\right)}{d s} e^{-\mathbb{B}_{s}\left(\tau_{r}\right)^{2}}\right) .
$$

From this lemma we obtain a second useful result:

Proposition 10. Let $\tau$ be a spectral cut for $\mathrm{\partial}_{0}$ and let $\theta \in \mathscr{C}^{\infty}(B ; \mathbb{R})$ be a smooth function with the property that null( $\left(\mathrm{\partial}_{0}+\theta\right)$ is a smooth vector bundle, $\tau(z)>\theta(z)$ and $\operatorname{spec}\left({\partial_{0, z}}\right) \cap(-\tau(z),-\theta(z))=\emptyset$ for each $z \in B$. Let us choose an homotopy $\tau_{r}$ between $\theta \equiv \tau_{0}$ and $\tau \equiv \tau_{1}$ such that $\theta \leqq \tau_{r} \leqq \tau$ and $\tau_{r}=\theta+r$ for $r \in[0, \bar{r}), \bar{r}<1$ small. Then

$$
\begin{aligned}
\widehat{\eta}_{\tau}= & \widehat{\eta}_{\theta}+\operatorname{Ch}\left(\operatorname{null}\left(\mathrm{\circlearrowright}_{0}+\theta\right), \nabla^{\text {null }}\right)+\int_{0}^{1} \operatorname{LIM}_{t \downarrow 0}\left(-\frac{t^{\frac{1}{2}}}{\sqrt{\pi}} \operatorname{STr}_{\mathrm{Cl}(1)}\left(\sigma \dot{\tau}_{r} e^{-\mathbb{B}_{t}\left(\tau_{r}\right)^{2}}\right)\right) d r \\
& +\int_{0}^{1} \operatorname{LIM}_{t \downarrow 0}\left(-d_{b} \int_{t}^{\infty} \operatorname{STr}_{\mathrm{Cl}(1)}\left(\sigma \dot{\tau}_{r} \frac{s^{\frac{1}{2}}}{\sqrt{\pi}} \frac{d \mathbb{B}_{s}\left(\tau_{r}\right)}{d s} e^{-\mathbb{B}_{s}\left(\tau_{r}\right)^{2}}\right) d s\right) d r .
\end{aligned}
$$

Proof. We integrate the formula of Lemma 4, first in $s$, from $t$ to $\infty$, and then in $r$, from $\varepsilon$ to $r=1$. Taking the regularized limits for small time we obtain

$$
\begin{aligned}
\widehat{\eta}_{\tau}= & \widehat{\eta}_{(\theta+\varepsilon)}+\int_{\varepsilon}^{1} \operatorname{LIM}\left(-\frac{t^{\frac{1}{2}}}{\sqrt{\pi}} \operatorname{STr}_{\mathrm{Cl}(1)}\left(\sigma \dot{\tau}_{r} e^{-\mathbb{B}_{t}\left(\tau_{r}\right)^{2}}\right)\right) d r \\
& +\int_{\varepsilon}^{1} \operatorname{LIM}_{t \downarrow 0}\left(-d_{b} \int_{t}^{\infty} \operatorname{STr}_{\mathrm{Cl}(1)}\left(\sigma \dot{\tau}_{r} \frac{s^{\frac{1}{2}}}{\sqrt{\pi}} \frac{d \mathbb{B}_{s}\left(\tau_{r}\right)}{d t} e^{-\mathbb{B}_{s}\left(\tau_{r}\right)^{2}}\right) d s\right) d r .
\end{aligned}
$$

Since from Sect. 16 of [18] it follows that

$$
\lim _{\varepsilon \rightarrow 0} \widehat{\eta}_{(\theta \pm \varepsilon)}=\widehat{\eta}_{\theta} \pm \operatorname{Ch}\left(\operatorname{null}\left(\partial_{0}+\theta\right), \nabla^{\text {null }}\right)
$$

the required formula follows from taking the limit as $\varepsilon \rightarrow 0$ in (7.14). To justify the existence of the limit in $\varepsilon$ of the last integral on the right-hand side we need to use the results of [4], and more precisely, Theorem 12, which gives the (improved) 
large time estimates of the heat kernel of the superconnection $\sigma\left(\varlimsup_{0}+\theta\right)+\mathbb{B}_{[1]}$. (Notice that since the term $\mathbb{B}_{[2]}$ in the Bismut superconnection is used only for the small time behaviour, we can assume that for $s$ large we are dealing precisely with $\left.s^{\frac{1}{2}} \sigma\left(ð_{0}+\tau\right)+\mathbb{B}_{[1]}.\right)$

From (7.9), (7.12) and the above lemma we obtain:

Proposition 11. Let ð be a family of Dirac operator on exact b-manifolds as in Sect. 1 and let us assume that for the boundary family the assumptions of Proposition 10 hold. Then if the null spaces of $ð(\tau)_{z}$ form a smooth vector bundle we have the equality of differential forms

$$
d \alpha_{b}(\tau)+d \gamma_{b}(\theta, \tau)+\operatorname{Ch}\left(\operatorname{null} ð(\tau), \nabla^{\text {null }}\right)=\operatorname{AS}(\theta)-\frac{1}{2} \widehat{\eta}_{\theta}-\frac{1}{2} \operatorname{Ch}\left(\operatorname{null}\left(\partial_{0}+\theta\right), \nabla^{\text {null }}\right)
$$

with $\alpha_{b}(\tau)$ fixed by (7.10) and

$$
\begin{aligned}
\gamma_{b}(\theta, \tau)= & \int_{0}^{1} \operatorname{LIM}_{t \downarrow 0}\left(\int_{t}^{\infty} \operatorname{STr}_{\mathrm{Cl}(1)}\left(\sigma \dot{\tau}_{r} \frac{s^{\frac{1}{2}}}{2 \sqrt{\pi}} \frac{d \mathbb{B}_{s}\left(\tau_{r}\right)}{d s} e^{-\mathbb{B}_{s}\left(\tau_{r}\right)^{2}}\right) d s\right) d r \\
& +\int_{0}^{1} \operatorname{LIM}_{t \downarrow 0} b-\operatorname{STr}\left(\frac{d \mathbb{A}_{t}\left(\tau_{r}\right)}{d r} e^{-\mathbb{A}_{t}\left(\tau_{r}\right)^{2}}\right) d r .
\end{aligned}
$$

As a particular case let us assume that the boundary family $ð_{0}$ has null space of constant dimension. Thus we can choose $\theta \equiv 0$.

If $\tau=u, u>0$ small and $\tau_{r}=r, r \in[0, u]$, then under the assumption that the null spaces of $\partial(u)_{z}$ are of constant dimension we obtain the fundamental formula

$$
\begin{aligned}
d \alpha_{b}(u)+d \gamma_{b}(u)+\operatorname{Ch}\left(\operatorname{null}(ð(u)), \nabla^{\text {null }}\right)= & \frac{1}{(2 \pi i)^{\frac{n}{2}}} \int_{M / B} \widehat{A}(M / B) \operatorname{Ch}^{\prime}(E) \\
& -\frac{1}{2} \widehat{\eta}-\frac{1}{2} \operatorname{Ch}\left(\operatorname{null}\left(\partial_{0}\right), \nabla^{\text {null }}\right)
\end{aligned}
$$

with $\alpha_{b}(u)$ and $\gamma_{b}(u)$ fixed by (7.10) and (7.17) respectively.

\section{Section 8. Hermitian Geometry and Surgery in the Constant Rank Case}

Let $ð$ be a family of Dirac operators as in Sect. 1. We now work under assumption (1.5):

The null spaces of the boundary family $ð_{0, z}, z \in B$, are of constant dimension.

Let $u \in \mathbb{R}, u>0$ and $u<\sigma_{0}$, with $\sigma_{0}$ less than the smallest positive eigenvalue of $ð_{0, z}$ for each $z \in B$. We consider the conjugated family

$$
ð(u)^{ \pm}=ð^{ \pm}+x^{\mp u}\left[ð^{ \pm}, x^{ \pm u}\right] .
$$

Since the boundary family of $\partial(u)$, viz. $\partial_{0}+u$, is by construction invertible, we can define as in Sect. 1 a determinant line bundle $\operatorname{det}_{b}(ð(u))$. For each $z \in B$ and each $u \in\left(0, \sigma_{0}\right)$ we have $\operatorname{null}\left({\delta_{z}}_{z}(u)\right)=\operatorname{null}\left({\varlimsup_{z}}_{z}, \Pi_{\geqq}\right)$. Thus there are canonical isomorphisms $S_{u}: \operatorname{det}_{b}(ð(u)) \rightarrow \operatorname{det}_{b}\left(ð, \Pi_{\geqq}\right)$. 
Moreover it should be recalled at this point that in the product case there is a natural isomorphism $\operatorname{det}_{b}\left(ð, \Pi_{\geqq}\right) \leftrightarrow \operatorname{det}_{\mathrm{APS}}\left(\widehat{\jmath}, \Pi_{\geqq}\right)$.

We are now interested to investigate the Hermitian geometry of $\operatorname{det}_{b}\left(ð, \Pi_{\geqq}\right)$. In the invertible case analyzed in Sect. 6 the fundamental result was that there exists a natural connection with curvature precisely equal to the 2-form piece of the righthand side of the family index formula. The family index formula for the index bundle $\operatorname{Ind}\left(ð, \Pi_{\geqq}\right)$follows from (7.18):

$$
\operatorname{Ch}\left(\operatorname{Ind}\left(ð, \Pi_{\geqq}\right)\right)=\frac{1}{(2 \pi i)^{\frac{n}{2}}} \int_{M / B} \widehat{A}(M / B) \mathrm{Ch}^{\prime}(E)-\frac{1}{2} \widehat{\eta}-\frac{1}{2} \operatorname{Ch}\left(\operatorname{null}\left(\mathrm{\partial}_{0}\right), \nabla^{\mathrm{null}}\right),
$$

and we are now looking for a connection on $\operatorname{det}_{b}\left(ð, \Pi_{\geqq}\right)$with curvature equal to the 2-form piece of the right-hand side of (8.3). We solve this problem on $\operatorname{det}_{b}(\partial(u))$. According to the results of Sects. 2,3 we can define a $b$-Quillen metric $\|\cdot\|_{b, u}$ and a Bismut-Freed connection ${ }^{b} \nabla^{\text {det, } u}$. More precisely we can choose the cover $\mathscr{U}=\left\{U_{\lambda}\right\}$ of Sect. 2 uniformly in $u$, for $u>0$ small. Then $\operatorname{det}_{b}(ð(u)) \uparrow$ $U_{\lambda}=\operatorname{det}\left(H_{[0, \lambda)}(u)\right)$ with $H_{[0, \lambda)}(u)$ equal to the direct sum of the eigenspaces of $\partial(u)$ corresponding to eigenvalues less than $\lambda$. We simply define the Quillen metric and the Bismut-Freed connection on $U_{\lambda}$ substituting in Definition (2.5) and (3.4) the family $ð(u)$ to the family $ð$ :

$$
\|\cdot\|_{b, u}=e^{-{ }^{b} \zeta^{\prime}\left(0, \partial^{-}(u) \partial^{+}(u), \lambda\right)}|\cdot|_{\lambda} \quad{ }^{b} \nabla^{\operatorname{det}, u}={ }^{b} \nabla^{\lambda, u}+\beta_{b, u}^{+}(\lambda)
$$

with $\nabla^{\lambda, u}, \beta_{b, u}^{+}(\lambda)$ defined as in (3.3), (3.2) but with $\partial(u)$ instead of $ð$.

Applying formula $(7.9)$ to $ð(u)_{(\lambda, \infty)}$ we get:

$$
d\left(\alpha_{b}(u, \lambda)\right)+\operatorname{Ch}\left(H_{[0, \lambda)}(u)\right)=\operatorname{AS}(u)-\frac{1}{2} \widehat{\eta}(u)
$$

with the Chern character computed with respect to the connection obtained by compressing $\mathbb{A}_{[1]}$ onto $H_{[0, \lambda)}(u)$. Proceeding as in Sect. 6 we get the equality of differential forms:

$$
\left({ }^{b} \nabla^{\operatorname{det}, u}\right)^{2}=\operatorname{AS}(u)_{[2]}-\frac{1}{2} \widehat{\eta}(u)_{[2]} .
$$

This is the local anomaly formula in the weighted case. Using the variational formulae of Sect. 7 we can improve (8.4) as follows. Formula (7.18) shows that if

$$
\begin{aligned}
\gamma_{b}(u)= & \int_{0}^{u} \operatorname{LIM}\left(\int_{t}^{\infty} \operatorname{STr}_{\operatorname{Cl}(1)}\left(\sigma \frac{s^{\frac{1}{2}}}{2 \sqrt{\pi}} \frac{d \mathbb{B}_{s}(r)}{d s} e^{-\mathbb{B}_{s}(r)^{2}}\right) d s\right) d r \\
& +\int_{0}^{u} \operatorname{LIM}_{t \downarrow 0} b-\operatorname{STr}\left(\frac{d \mathbb{A}_{t}(r)}{d r} e^{-\mathbb{A}_{t}(r)^{2}}\right) d r,
\end{aligned}
$$

then on $U_{\lambda}$

$$
\begin{aligned}
d \alpha_{b}(u, \lambda)+d \gamma_{b}(u)+\operatorname{Ch}\left(H_{[0, \lambda)}(u)\right)= & \frac{1}{(2 \pi i)^{\frac{n}{2}}} \int_{U_{\lambda} / B} \widehat{A}(M / B) \mathrm{Ch}^{\prime}(E) \\
& -\frac{1}{2} \widehat{\eta}-\frac{1}{2} \operatorname{Ch}\left(\operatorname{null}\left({ }_{0}\right), \nabla^{\text {null }}\right)
\end{aligned}
$$


as differential forms on $U_{\lambda}$. Thus if we define a perturbed Bismut-Freed connection as

$$
{ }^{b} \widetilde{\nabla}^{\operatorname{det}, u}={ }^{b} \nabla^{\operatorname{det}, u}+\gamma_{b}(u)_{[1]},
$$

we finally get

$$
\begin{aligned}
& \left({ }^{b} \widetilde{\nabla}^{\operatorname{det}, u} \uparrow U_{\lambda}\right)^{2}=\left({ }^{b} \nabla^{\lambda, u}+\beta_{b, u}^{+}(\lambda)\right)^{2}+d \gamma_{b}(u)_{[1]} \\
& \quad=d\left(\alpha_{b}(u, \lambda)_{[1]}\right)+\operatorname{Ch}\left(H_{[0, \lambda)}(u)\right)_{[2]}+d \gamma(u)_{[1]} \\
& \quad=\left[\frac{1}{(2 \pi i)^{\frac{n}{2}}} \int_{U_{\lambda} / B} \widehat{A}(M / B) \operatorname{Ch}^{\prime}(E)-\frac{1}{2} \widehat{\eta}-\frac{1}{2} \operatorname{Ch}\left(\operatorname{null}\left(ð_{0}\right), \nabla^{\text {null }}\right)\right]_{[2]} .
\end{aligned}
$$

Note that the above arguments can be carried over for a negative weight as well. In this case, using $(7.15)$, we obtain a perturbed Bismut-Freed connection ${ }^{b} \widetilde{\nabla}^{(\text {det },-u)}$ with curvature

$\left({ }^{b} \widetilde{\nabla}^{(\mathrm{det},-u)}\right)^{2}=\left[\frac{1}{(2 \pi i)^{\frac{n}{2}}} \int_{M / B} \widehat{A}(M / B) \mathrm{Ch}^{\prime}(E)-\frac{1}{2} \widehat{\eta}+\frac{1}{2} \operatorname{Ch}\left(\operatorname{null}\left(\mathrm{\delta}_{0}\right), \nabla^{\mathrm{null}}\right)\right]_{[2]}$.

Summarizing, by fixing any $u \in\left(0, \sigma_{0}\right)$ and considering the canonical identification $\operatorname{det}_{b}\left(ð, \Pi_{\geqq}\right) \leftrightarrow \operatorname{det}_{b}(ð(u))$ we can endow the line bundle $\operatorname{det}_{b}\left(ð, \Pi_{\geqq}\right)$ with a $b$-Quillen metric and a Bismut-Freed connection ${ }^{b} \nabla^{\text {det }}$. Moreover we can perturb ${ }^{b} \nabla^{\text {det }}$ and define a connection ${ }^{b} \widetilde{\nabla}^{\text {det }}$ with curvature equal to the 2 -form piece of (8.6). We have proved the following

Theorem 2. Let ð be a family of Dirac operators on manifolds with boundary as in Sect. 1. Let us assume the boundary family $\mathrm{\partial}_{0}$ to have null space of constant dimension in $z \in B$. Let us endow the resulting smooth vector bundle null( $\left.\partial_{0}\right)$ with the connection $\nabla^{\text {null }}$ obtained by compressing onto null $\left(\mathrm{\partial}_{0}\right)$ the 1-form piece of the Bismut superconnection. Let $\operatorname{det}_{b}\left(ð, \Pi_{\geqq}\right)$be the determinant line bundle associated to $\partial$ and the spectral section $\Pi_{\geqq}$defined by the spectral projection associated to the non-negative eigenvalues of $\bar{\partial}_{0}$. Then

(i) There exists a connection ${ }^{b} \widetilde{\nabla}^{\mathrm{det}}$ on $\operatorname{det}_{b}\left(ð, \Pi_{\geqq}\right)$, which is a natural perturbation of the Bismut-Freed connection, with curvature

$$
\left({ }^{b} \widetilde{\nabla}^{\mathrm{det}}\right)^{2}=\left[\frac{1}{(2 \pi i)^{\frac{n}{2}}} \int_{M / B} \widehat{A}(M / B) \mathrm{Ch}^{\prime}(E)-\frac{1}{2} \widehat{\eta}-\frac{1}{2} \operatorname{Ch}\left(\operatorname{null}\left(\mathrm{\partial}_{0}\right), \nabla^{\mathrm{null}}\right)\right]_{[2]} .
$$

(ii) If the metrics are of product type near the boundary and if $\left(\widehat{\varnothing}, \Pi_{\geqq}\right)$denotes the Atiyah-Patodi-Singer family in the incomplete setting then there exists a natural explicit isomorphism

$$
T: \operatorname{det}_{\mathrm{APS}}\left(\widehat{\partial}, \Pi_{\geqq}\right) \rightarrow \operatorname{det}_{b}\left(ð, \Pi_{\geqq}\right) .
$$

Finally let us consider the surgery problem of Sect. 4 when we relax assumption (4.4) and only assume:

the null spaces of the family $ð_{H}$ are of constant dimension. 
On both $\bar{M}^{0}$ and $\bar{M}^{1}$ we can consider conjugated families of the type considered in (8.2) with $u$ either positive or negative but in any case chosen in the interval $\left(-\sigma_{0}, \sigma_{0}\right)$ with $\sigma_{0}>0$ less than the square root of the smallest positive eigenvalue of the family $\partial_{H}^{2}$.

By assumption (8.10) these families are Fredholm on unweighted Sobolev spaces. Thus there are well defined determinant bundles defined as in Sect. 1.

Let $\partial$ be the original family associated with the closed fibration $(\psi: M \rightarrow B$, $\left.g_{M / B}\right)$ and for $\varepsilon \in\left(0, \varepsilon_{0}\right]$ and $u>0$ let us consider the family of Dirac operators

$$
\partial^{ \pm}(\varepsilon, u)=\left(x^{2}+\varepsilon^{2}\right)^{\mp \frac{u}{2}} \partial^{ \pm}(\varepsilon)\left(x^{2}+\varepsilon^{2}\right)^{ \pm \frac{u}{2}}
$$

with $\partial(\varepsilon)$ as in Sect. 4. Clearly for each $z \in B$ the operator $\partial_{z}(\varepsilon, u)$ is an element of the surgery calculus of $M_{z}: ð_{z}(\varepsilon, u) \in \operatorname{Diff}_{s}^{1}\left(M_{z} ; E\right)$. Moreover $ð_{z}(0, u)$ is a conjugated operator $\mathrm{\partial}_{z, \bar{M}}(u) \in \operatorname{Diff}_{b}^{1}\left(\bar{M}_{z}, E\right)$ of the type considered above.

Using the identifications of Sect. 4 we obtain for the limit operators the analogues of (4.2), (4.3), namely:

$$
\begin{gathered}
L_{0}^{\mp} \cdot\left(\partial^{ \pm}(0, u)\right)_{\bar{M}^{1}} \cdot\left(L_{0}^{ \pm}\right)^{-1}= \pm x \frac{\partial}{\partial x}+{\varlimsup_{H}}+u, \\
L_{0}^{\mp} \cdot\left(\partial^{ \pm}(0, u)\right)_{\bar{M}^{0}} \cdot\left(L_{0}^{ \pm}\right)^{-1}= \pm y \frac{\partial}{\partial y}+ð_{(-H)}-u \quad \text { with } y=-x .
\end{gathered}
$$

We shall denote by ${\partial_{\bar{M}}}^{0}(-u), \partial_{\bar{M}^{1}}(u)$ the $b$-families induced by $\partial_{\bar{M}}(u)$ on $\bar{M}^{0} \rightarrow B$, $\bar{M}^{1} \rightarrow B$ respectively. Notice that by considering $(8.11)$ we have again reduced the constant rank case to the invertible case.

We now concentrate on $\operatorname{det}(\varepsilon, u)$. First observe that we can equip the determinant bundles $\operatorname{det}(ð(\varepsilon, u)), \operatorname{det}_{b}\left(\delta_{\bar{M}^{0}}(-u)\right), \operatorname{det}_{b}\left(\delta_{\bar{M}^{1}}(u)\right)$ with Quillen metrics and Bismut-Freed connections $\nabla^{\text {det, } \varepsilon, u},{ }^{b} \nabla_{\bar{M}^{0}}^{(\text {det, }-u)},{ }^{b} \nabla_{\bar{M}^{1}}^{(\text {det }, u)}$ with curvatures $R_{\operatorname{det}}(\varepsilon, u)$, $R_{\operatorname{det}_{b}}^{0}(-u), R_{\operatorname{det}_{b}}^{1}(u)$ respectively. By the results of Sect. 4 we have a natural isomorphism $\operatorname{det}(ð(\varepsilon, u)) \leftrightarrow \operatorname{det}_{b}\left(\varlimsup_{\bar{M}}\right) \equiv \operatorname{det}_{b}\left({\partial_{\bar{M}}}(-u)\right) \otimes \operatorname{det}_{b}\left(ð_{\bar{M}^{1}}(u)\right)$. Let us denote by $\Pi_{\geqq}$the spectral projection associated to the non-negative eigenvalues of $ð_{H}$. Using (8.12) we easily obtain canonical identifications $\operatorname{det}_{b}\left(\partial_{\bar{M}^{1}}(u)\right) \leftrightarrow \operatorname{det}_{b}\left(\partial_{\bar{M}^{1}}, \Pi_{\geqq}\right)$, $\operatorname{det}_{b}\left(\partial_{\bar{M}^{0}}(-u)\right) \leftrightarrow \operatorname{det}_{b}\left({\partial_{\bar{M}} 0}_{1}, \Pi_{<}\right), u>0$. Thus there exist natural isomorphisms

$$
S(\varepsilon, u): \operatorname{det}(ð(\varepsilon, u)) \rightarrow \operatorname{det}_{b}\left(ð_{\bar{M}^{0}}, \Pi_{<}\right) \otimes \operatorname{det}_{b}\left({\partial_{\bar{M}}}, \Pi_{\geqq}\right) .
$$

Moreover as $\varepsilon \downarrow 0$ we have

$$
\begin{gathered}
R_{\operatorname{det}}(\varepsilon, u) \rightarrow R_{\operatorname{det}_{b}}^{0}(-u)+R_{\operatorname{det}_{b}}^{1}(u), \\
\operatorname{hol}_{\gamma}\left(\nabla^{\operatorname{det}, \varepsilon, u}\right) \rightarrow \operatorname{hol}_{\gamma}\left({ }^{b} \nabla_{\bar{M}^{0}}^{(\operatorname{det},-u)}\right) \cdot \operatorname{hol}_{\gamma}\left({ }^{b} \nabla_{\bar{M}^{1}}^{(\operatorname{det}, u)}\right) \quad \forall \gamma \in \operatorname{Map}\left(\mathbb{S}^{1}, B\right) .
\end{gathered}
$$

Although these results are interesting in their own right it is clear that these are not the right connection to consider, the reason being that they are associated to Dirac families which are not defined by Hermitian Clifford connections. In particular the small time behaviour of the superconnection heat kernel is not well behaved and the curvatures $R_{\operatorname{det}}(\varepsilon, u), R_{\operatorname{det}_{b}}^{0}(-u), R_{\operatorname{det}_{b}}^{1}(u)$ are not easily computable (they are in fact given by regularized limits). We remedied this problem for weighted operators on manifolds with boundary in the first part of this section when we perturbed the Bismut-Freed connection, obtaining a connection on $\operatorname{det}_{b}(ð(u)) \leftrightarrow \operatorname{det}_{b}\left(ð, \Pi_{\geqq}\right)$with curvature as computable as possible (see Theorem 2 ). We now apply this procedure 
to $\delta_{\bar{M}^{0}}(-u)$ and $\delta_{\bar{M}^{1}}(u)$ obtaining connections ${ }^{b} \widetilde{\nabla}_{\bar{M}^{0}}^{(\mathrm{det},-u) b} \widetilde{\nabla}_{\bar{M}^{1}}^{(\mathrm{det}, u)}$. Similarly we can perturb the connection $\nabla^{\text {det, } \varepsilon, u}$ by the one form

$$
\gamma(\varepsilon, u)_{[1]}=\int_{0}^{u} \operatorname{LIM}_{t \downarrow 0} \operatorname{STr}\left(\frac{d \mathbb{A}_{t}(\varepsilon, r)}{d r} e^{-\mathbb{A}_{t}^{2}(\varepsilon, r)}\right)_{[1]} d r,
$$

thus defining $\widetilde{\nabla}^{\text {det, } \varepsilon, u}=\nabla^{\text {det, } \varepsilon, u}+\gamma(\varepsilon, u)_{[1]}$ with curvature equal to $R_{\operatorname{det}}(\varepsilon)$ of Sect. 4 . Applying the heat surgery calculus as in Proposition 6 we obtain

$$
\lim _{\varepsilon \rightarrow 0} \gamma(\varepsilon, u)_{[1]}+\log \varepsilon \cdot d \xi(u)=\gamma_{b, \bar{M}}(u)_{[1]}
$$

with $\xi(u) \in \mathscr{C}^{\infty}(B)$ given in term of the heat kernel of $\partial_{\widetilde{H}}$. Thus as $\varepsilon \downarrow 0$ we have the convergence of the push-forward of

to

$$
\begin{gathered}
\widetilde{\nabla}^{\mathrm{det}, \varepsilon, u}+\log \varepsilon \cdot d\left(\frac{1}{2} \zeta^{\prime}\left(0,\left(\mathrm{\partial}_{\widetilde{H}}+u\right)^{2}\right)+\xi(u)\right) \\
{ }^{b} \widetilde{\nabla}_{\bar{M}}^{(\mathrm{det}, u)} \equiv{ }^{b} \widetilde{\nabla}_{\bar{M}^{0}}^{(\mathrm{det},-u)} \otimes \mathrm{Id}+\mathrm{Id} \otimes{ }^{b} \widetilde{\nabla}_{\bar{M}^{1}}^{(\mathrm{det}, u)} .
\end{gathered}
$$

It is clear as in Sect. 4 that the extra-term, being exact, does not contribute to the computation of the curvature and of the holonomy. We can finally state our main result about surgery:

Theorem 3. Let $\left(\psi: M \rightarrow B, g_{M / B}\right)$ be a closed Riemannian fibration and let $\succsim \in$ $\operatorname{Diff}_{\phi}^{1}(M ; E)$ denote a family of Dirac-type operators associated to a Hermitian Clifford connection. Let $H$ be a disconnecting fibering hypersurface in $M$ with defining function $x \in \mathscr{C}^{\infty}(M)$ as in Sect. 4 and let us make assumption (8.10). For $\varepsilon \in\left(0, \varepsilon_{0}\right]$ consider the family $\partial(\varepsilon)$ associated to the family of Riemannian metrics $g(\varepsilon)=|d x|^{2} /\left(x^{2}+\varepsilon^{2}\right)+g_{M / B}$. Let $u>0$ be less than $\sigma_{0}$ in turn less than

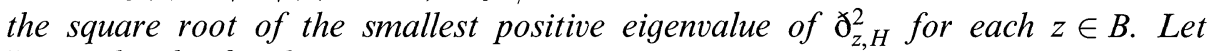
$ð(\varepsilon, u)$ be the family $(8.11)$.

(i) The determinant line bundles $\operatorname{det}(ð)$ and $\operatorname{det}(ð(\varepsilon, u))$ are isomorphic.

(ii) Let $\bar{M}=\bar{M}^{0} \sqcup \bar{M}^{1}$ be the b-fibration associated to the b-metric $g(0)$ and let $\searrow_{\bar{M}}=\searrow_{\bar{M}^{0}} \oplus{\partial_{\bar{M}}}$ be the limit operator. Then for each fixed $\varepsilon \in\left(0, \varepsilon_{0}\right], u \in$ $\left(0, \sigma_{0}\right)$ there exists a natural isomorphism

$$
S(\varepsilon, u): \operatorname{det}(ð(\varepsilon, u)) \rightarrow \operatorname{det}_{b}\left(\partial_{\bar{M}^{0}}, \Pi_{<}\right) \otimes \operatorname{det}_{b}\left(\partial_{\bar{M}^{1}}, \Pi_{\geqq}\right)
$$

and thus an isomorphism $S: \operatorname{det}(ð) \rightarrow \operatorname{det}_{b}\left(\varlimsup_{\bar{M}^{0}}, \Pi_{<}\right) \otimes \operatorname{det}_{b}\left(\varlimsup_{\bar{M}^{1}}, \Pi_{\geqq}\right)$Fix $u \in$ $\left(0, \sigma_{0}\right)$. Then we equip the three line bundles appearing in (8.14) with perturbed Bismut-Freed connections $\widetilde{\nabla}^{\mathrm{det}, \varepsilon}{ }^{b} \widetilde{\nabla}^{\mathrm{det}, 0},{ }^{b} \widetilde{\nabla}^{\mathrm{det}, 1}$ respectively in such a way that for the curvatures and the holonomies the following formulae hold:

$$
\begin{gathered}
\lim _{\varepsilon \rightarrow 0}\left(\widetilde{\nabla}^{\text {det, }, \varepsilon}\right)^{2}=\left({ }^{b} \widetilde{\nabla}^{\text {det, }, 0}\right)^{2}+\left({ }^{b} \widetilde{\nabla}^{\text {det, } 1}\right)^{2}, \\
\lim _{\varepsilon \rightarrow 0} \operatorname{hol}_{\gamma}\left(\widetilde{\nabla}^{\text {det, }, \varepsilon}\right)=\operatorname{hol}_{\gamma}\left({ }^{b} \widetilde{\nabla}^{\text {det }, 0}\right) \cdot \operatorname{hol}_{\gamma}\left({ }^{b} \widetilde{\nabla}^{\text {det, } 1}\right) \quad \forall \gamma \in \operatorname{Map}\left(\mathbb{S}^{1}, B\right) .
\end{gathered}
$$

Once again the additivity of the curvatures can also be proved by making use of the local anomaly formula in the closed case together with (8.7), (8.8) (see also (8.12)). 
Acknowledgements. It is a pleasure to thank Xianzhe Dai, Andrew Hassel, John Lott, Rafe Mazzeo, Richard Melrose and Simon Scott for many illuminating conversations.

\section{References}

1. Atiyah, M.F., Singer, I.M.: Dirac operators coupled to vector potentials. Proc. Nat. Acad. Sci. 81, 2596-2600 (1984)

2. Berline, N., Getzler, E., Vergne, M.: Heat kernels and Dirac operators. Berlin, Heidelberg, New York: Springer, 1992

3. Berline, N., Vergne, M.: A proof of the Bismut local theorem for a family of Dirac operators. Topology 26, 435-463 (1987)

4. Berline, N., Vergne, M.: The equivariant Chern character and the index of $G$-invariant operators. CIME proceedings. Lectures notes in Mathematics, Vol. 1565 (1993)

5. Bismut, J.-M.: The index theorem for families of Dirac operators: Two heat equation proofs. Invent. Math. 83, 91-151 (1986)

6. Bismut, J.-M., Cheeger, J.: Families index for manifolds with boundary superconnections and cones I. J. Funct. Anal. 89, 313-363 (1990)

7. Bismut, J.-M., Cheeger, J.: Families index for manifolds with boundary superconnections and cones II. J. Funct. Anal. 90, 306-354 (1990)

8. Bismut, J.-M., Cheeger, J.: $\eta$-invariants and their adiabatic limits. JAMS 2, 33-70 (1989)

9. Bismut, J.-M., Cheeger, J.: Remarks on the index theorem for families of Dirac operators on manifolds with boundary. In: "Differential Geometry", B. Lawson, K. Teneblat, (eds), Longman Scientific, 1992

10. Bismut, J.-M., Freed, D.S.: The analysis of elliptic families: Metrics and connections on determinant bundles. Commun. Math. Phys. 106, 159-176 (1986)

11. Bismut, J.-M., Freed, D.S.: The analysis of elliptic families: Dirac operators, eta invariants and the holonomy theorem of Witten. Commun. Math. Phys. 107, 103-163 (1986)

12. Bunke, U.: On the gluing problem for the eta-invariant. J. Diff. Geom. 41, 397-448 (1995)

13. Cheeger, J.: Eta invariants, the adiabatic approximation and conical singularities. J. Diff. Geom. 26, 175-221 (1987)

14. Freed, D.S.: Determinants, torsion and strings. Commun. Math. Phys. 107, 1-18 (1896)

15. Hassel, A., Mazzeo R., Melrose, R.: Analytic surgery and the accumulation of eigenvalues. To appear in Commun. Anal. Geom.

16. Mazzeo, R., Melrose, R.: Analytic surgery and the eta invariant. Geom. Funct. Anal. 5, 14-75 (1995)

17. Melrose, R.B.: The Atiyah-Patodi-Singer Index Theorem. A. and K. Peters, 1993

18. Melrose, R.B., Piazza, P.: Families of Dirac operators, boundaries and the $b$-calculus. To appear in J. Diff. Geom.

19. Melrose, R.B., Piazza, P.: An index theorem for families of Dirac operators on odd-dimensional manifolds with boundary. To appear in J. Diff. Geom.

20. Müller, W.: The eta invariant on manifolds with boundary. J. Diff. Geom. (1995)

21. Piazza, P.: $K$-theory and index theory on manifolds with boundary. Thesis MIT, May 1991

22. Piazza, P.: On the index of elliptic operators on manifolds with boundary. J. Funct. Anal. 117, 308-359 (1993)

23. Quillen, D.: Determinants of Cauchy-Riemann operators over a Riemann surface. Funct. Anal. Appl. 14, 31-34 (1985)

24. Reed, N., Simon, B.: Methods of modern mathematical physics. New York: Acad. Press, 1972

25. Scott, S.G.: Determinants of Dirac Boundary Value Problems over Odd-Dimensional Manifolds. Commun. Math. Phys., Vol. 173 (1995)

26. Singer, I.M.: The eta invariant and the index. Mathematical aspects of string theory. S.T. Yau, (ed.) Singapore: World Scientific, 1987

27. Witten, E.: Global gravitational anomalies. Commun. Math. Phys. 100, 197-229 (1985) 